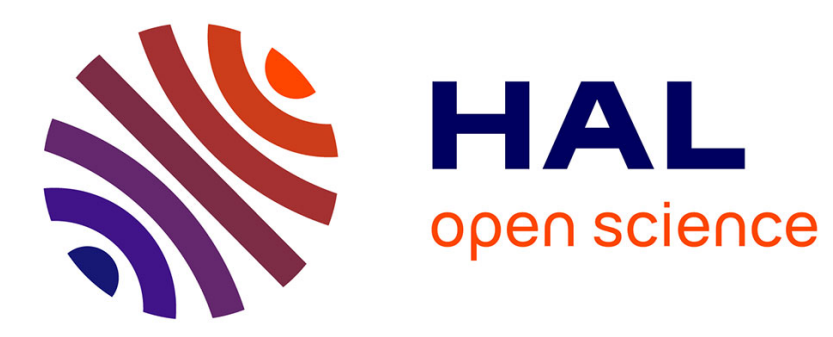

\title{
Quelques aspects statistiques des processus d'aimantation dans les corps ferromagnétiques. Cas du déplacement d'une seule paroi de Bloch à $180^{\circ}$ dans un milieu monocristallin aléatoirement perturbé
}

R. Vergne, J.C. Cotillard, J.L. Porteseil

\section{To cite this version:}

R. Vergne, J.C. Cotillard, J.L. Porteseil. Quelques aspects statistiques des processus d'aimantation dans les corps ferromagnétiques. Cas du déplacement d'une seule paroi de Bloch à $180^{\circ}$ dans un milieu monocristallin aléatoirement perturbé. Revue de Physique Appliquée, 1981, 16 (9), pp.449476. 10.1051/rphysap:01981001609044900 . jpa-00244939

HAL Id: jpa-00244939

https://hal.science/jpa-00244939

Submitted on 1 Jan 1981

HAL is a multi-disciplinary open access archive for the deposit and dissemination of scientific research documents, whether they are published or not. The documents may come from teaching and research institutions in France or abroad, or from public or private research centers.
L'archive ouverte pluridisciplinaire HAL, est destinée au dépôt et à la diffusion de documents scientifiques de niveau recherche, publiés ou non, émanant des établissements d'enseignement et de recherche français ou étrangers, des laboratoires publics ou privés. 


\title{
Quelques aspects statistiques des processus d'aimantation dans les corps ferromagnétiques. Cas du déplacement d'une seule paroi de Bloch à $180^{\circ}$ dans un milieu monocristallin aléatoirement perturbé
}

\author{
R. Vergne, J. C. Cotillard et J. L. Porteseil \\ C.N.R.S.. Laboratoire Louis-Néel (*). 166 X. 38042 Grenoble Cedex, France \\ (Reçu le 29 septembre 1980, révisé le 26 mai 1981, accepté le 4 juin 1981)
}

\begin{abstract}
Résumé. - Cet exposé étant destiné à un public de non-spécialistes, nous avons d'abord rappelé comment s'aimante un matériau ferromagnétique et ce qu'est un processus d'aimantation. Puis, nous avons envisagé le cas le plus simple qu'il soit possible de réaliser expérimentalement qui est celui d'une paroi de Bloch à $180^{\circ}$ unique, se déplaçant dans un milieu monocristallin aléatoirement perturbé. Pour cela, nous avons utilisé un cadre de $\mathrm{Fe}$-Si dont les côtés sont parallèles aux axes de facile aimantation. Le problème a un double caractère aléatoire. Le premier est dû à l'existence de défauts, répartis au hasard, qui gênent le déplacement de la paroi; le second tient à l'agitation thermique. Son action sur les moments élémentaires est génératrice d'un champ aléatoire fluctuant, pouvant s'ajouter ou se retrancher au champ appliqué et permettre de ce fait à la paroi le franchissement d'obstacles que le champ seul n'aurait pas permis. Même dans un cas aussi idéalement simple, les mécanismes réels ont une certaine complexité.
\end{abstract}

Pour une excursion de champ donnée, on peut observer deux modes de déplacement irréversibles de la paroi en fonction de la vitesse qui lui est imposée, ou de la température. La paroi peut se déplacer en bloc comme une membrane rigide, ses déplacements sont alors toujours supérieurs à son épaisseur. Le bruit Barkhausen est celui d'un régime de grands sauts. Le modèle de la fonction potentiel s'applique : il est possible de reconstituer $V(x)$, de déterminer la statistique des pentes aux points d'inflexion, leur corrélation et de montrer que pour des déplacements supérieurs à environ dix fois l'épaisseur de la paroi, on perd la propriété de conservativité. La paroi peut également se déplacer localement par petits morceaux en mettant à profit ses degrés de liberté interne. C'est le régime des petits sauts de Barkhausen. Le modèle de la fonction potentiel ne s'applique plus tel quel.

Le passage de l'un à l'autre régime peut s'effectuer à température constante en augmentant la vitesse de déplacement de la paroi. On observe d'abord un régime de petits sauts indépendants; pour une première vitesse critique, apparaissent des événements corrélés : c'est le régime des grands sauts. En continuant d'augmenter la vitesse, il apparaît un nouveau régime de petits sauts. La première transition a le caractère d'une instabilité. La seconde présente des analogies avec l'apparition de la turbulence en hydrodynamique. On observe les mêmes phénomènes à vitesse constante en faisant décroître la température.

L'évolution du cycle rectangulaire élémentaire en fonction du nombre de cycles décrits montre que le système s'adapte aux contraintes qui lui sont imposées. L'ensemble de ces faits s'éclaire d'un jour nouveau si l'on remarque que le système étudié est non linéaire, doué d'hystérésis et hors d'équilibre. Après un bref rappel du travail de Bonnet, qui nous a permis d'en dégager les hypothèses physiques, nous avons fait une analyse critique du modèle de la fonction potentiel. Nous avons enfin décrit un autre aspect des phénomènes à partir de l'étude de la force exercée par les défauts du réseau sur la paroi. Nous avons pu aussi introduire la notion d'homothétie interne dans les déplacements d'une paroi de Bloch.

L'étude de l'influence des fluctuations thermiques sur le déplacement d'une paroi de Bloch, dans le cas particulier où celle-ci est soumise à un champ voisin du champ coercitif, c'est-à-dire à une force presque suffisante pour qu'elle se décroche de tous les obstacles, nous a permis de préciser leur rôle, certaines grandeurs qui leur sont attachées (échelles de temps et d'espace caractérisant les processus d'aimantation, volume d'activation), des caractéristiques de la paroi (flexibilité) ou des imperfections du cristal (obstacles). Cette dernière notion nous a conduits à généraliser le concept de fonction potentiel au cas de la paroi flexible. Dès lors, on ne peut pas considérer l'agitation thermique comme une petite perturbation, mais comme un paramètre physique essentiel que doit prendre en compte tout modèle réaliste de déplacement de paroi.

Des méthodes statistiques simples appliquées aux séquences de sauts Barkhausen nous ont permis de tirer des conclusions physiques intéressantes sur les mécanismes d'aimantation. Mais, quoique le bruit Barkhausen soit toujours le reflet de ces mécanismes, il paraît extrêmement difficile, dans un cas plus complexe comme celui d'un polycristal, de remonter de ses caractéristiques aux causes qui l'ont produit. Il nous semble, sur deux points au

(*) Associé à l’Université Scientifique et Médicale de Grenoble. 
moins, qu'il soit possible d'utiliser des méthodes statistiques plus élaborées que celles que nous avons employées. Le premier point étant la recherche d'une ressemblance entre séquences de sauts correspondant à des déplacements de la paroi d'amplitude de plus en plus grande. On pourrait ainsi avoir des informations sur la manière dont on perd la propriété de conservativité. Le second est l'élaboration d'un modèle statistique des séquences de sauts Barkhausen à partir des résultats expérimentaux.

Abstract. - Since this paper aims to be readable by a non-specialist, we recall in the first part the essential features of magnetization processes in a ferromagnet. Then we consider the simplest possible experimental situation, which is the case of a single $180^{\circ}$ Bloch wall moving in a crystalline medium affected by random imperfections. For this purpose, we have studied a frame of $\mathrm{Fe}-\mathrm{Si}$, the edges of which are parallel to the easy axes of the crystal. The random character of the problem is twofold : firstly, the motion of the wall is hindered by lattice defects which are distributed at random. Secondly, the thermal motion of atomic moments generates a fluctuating field which is superimposed to the applied field and allows the wall to overcome obstacles at random instants.

Even in such a simple situation, the phenomena are rather complex. For a given range of fields, two types of irreversible wall motion can be observed, depending on the wall velocity and the temperature. The wall can jump like a rigid membrane; then its displacements are, at least, of the same order of magnitude as its thickness. The Barkhausen noise corresponds to a regime of large jumps. A model of potential function can be applied : it is possible to reconstitute $V(x)$, to determine the distribution of inflexion slopes and their correlation, and to show that the property of conservativity disappears when the displacement of the wall exceeds about 10 times its thickness. The wall can also behave like a flexible body and move by localized deformations. This behaviour results in a regime of small Barkhausen jumps. The model of the potential function is no longer valid.

The transition between the two regimes can take place at a given temperature if the velocity of the wall increases. Onc observes firstly a regime of small, independent jumps. At a first critical velocity, correlated events appear : this is the regime of large jumps. If the velocity further increases, a new regime of small jumps appears. The first transition has the character of an instability, the second one has some analogies with the onset of turbulence in fluid dynamics. The same succession of phenomena can be observed at a constant velocity by lowering the temperature.

The modifications of an elementary rectangular loop when successive cycles are described show that the system evolves in a way which depends on the external contraints. Those results can be interpreted by remarking that the system is non-linear, hysteretic and out of equilibrium.

After a recall of the work of Bonnet and a short analysis of its physical background, we present a critical study of the model of the potentiel function. Last, we point out another interesting feature of the force exerted by the lattice defects on the wall and we introduce the concept of self-similarity in Bloch wall motion.

A study of the influence of thermal activation on the motion of a Bloch wall in the peculiar case of an applied field close to the coercive force, that is to say almost sufficient for the wall to overcome all the obstacles, enabled us to precise the role of pinning centers, some physical quantities (time and space scales of magnetization processes, activation volume) and characteristics of the wall (flexibility) and the lattice defects (obstacles). The latter concept led us to generalize the idea of potential function to the case of a flexible wall. It turns out that thermal fluctuation should not be regarded as a small perturbation, but as an essential physical parameter which has to be taken into account in any realistic model of wall motion.

Simple statistical methods applied to sequences of Barkhausen jumps afford an interesting insight on the physics of magnetization processes. But, although the Barkhausen noise always reflects those phenomena, it seems exceedingly difficult, in the more complex case of a polycrystal, to extract from the Barkhausen noise precise information on the physical processes involved. Two problems at least seem to deserve the application of more sophisticated statistical methods. The first one is the search for a likeness between sequences of jumps generated by successive displacements of the wall of increasing magnitudes. Such a study might give useful information on the progressive disappearance of the property of conservativity. The second problem consists of elaborating a statistical model of Barkhausen noise. These two points might initiate an interesting cross-disciplinary work in the fields of random phenomena and magnetism.

Avant-propos. - Pourquoi écrire un article ayant trait aux processus d'aimantation essentiellement destiné aux physiciens s'intéressant aux méthodes et aux applications du traitement du signal. Il y a à cela plusieurs raisons, La première est le caractère aléatoire des phénomènes étudiés. Des méthodes statistiques simples comme l'établissement d'histogrammes ou des mesures classiques telles que corrélation, densité spectrale énergétique, nous ont permis d'obtenir des informations intéressantes sur la physique des phénomènes étudiés.

Il y a également le fait que de nombreux spécialistes des phénomènes aléatoires ont étudié le bruit Barkhausen dans diverses substances ferromagnétiques. Il a en général pour origine les déplacements irréversibles des parois sous l'influence du champ magnétique appliqué. Cette étude est consacrée à un cas très simple, celui d'une paroi de Bloch unique se déplaçant dans un milieu aléatoirement perturbé. Il est réalisable à partir d'un cadre monocristallin de fer-silicium convenablement taillé. On peut ainsi dégager les caractères spécifiques du déplacement de la paroi. Un modèle de "bruit Barkhausen" dans un cas complexe, comme celui d'un polycristal ferromagnétique, 
par exemple du fer pur ou de l'acier, ne peut ignorer les caractéristiques du cas élémentaire.

Enfin, cette étude permet de suggérer quelques problèmes plus spécifiques de traitement de signal. Nous souhaitons qu'elle soit un point de départ pour un travail pluridisciplinaire entre les spécialistes des phénomènes aléatoires et des processus d'aimantation.

Cet exposé résume un ensemble de travaux parus par ailleurs ou en cours de publication $\left({ }^{1}\right)$.

Comme il est destiné à un public de non-spécialistes, nous avons dû rappeler comment s'aimante un matériau ferromagnétique. Le lecteur intéressé par ce problème se reportera utilement aux références $[1,2]$, alors que le lecteur averti passera directement à la seconde partie.

\section{L'aimantation d'un monocristal ferromagnétique.} - 1.1 Le MODèle MiCROSCOPIQUE. - On peut concevoir à l'échelle atomique un corps ferromagnétique comme un assemblage régulier de moments magnétiques localisés. A chacun des ions ou des atomes qui composent le cristal est alors attaché un moment magnétique caractéristique de sa structure électronique. Des influences extérieures comme l'application d'un champ magnétique ou l'agitation thermique influent de façon négligeable sur celle-ci et ne peuvent provoquer que des rotations des moments. Les propriétés magnétiques du cristal : aimantation à saturation, hystérésis, aimantation rémanente sont le reflet des rotations des moments élémentaires qui le composent.

1.2 Les Différentes espèces D’ÉNERGIE. - Pour comprendre comment s'orientent les moments élémentaires, il est nécessaire de faire un bilan des différentes sortes d'énergies mises en jeu. Nous les classerons par ordre d'importance décroissante.

1.2.1 L'interaction d'échange responsable du ferromagnétisme. - L'interaction d'échange tend à aligner parallèlement les moments magnétiques voisins. $\mathrm{Si}$ elle agissait seule, les $N$ moments élémentaires seraient parfaitement alignés et le moment résultant du cristal serait le moment à saturation $\mathbf{J}_{\mathbf{S}}$.

( $\left.{ }^{1}\right)$ Remarque : Ce travail n'a aucun caractère exhaustif. Nous avons volontairement passé sous silence le cas du déplacement de la paroi dans un isolant, bien que le bruit de Barkhausen ait été étudié dans de telles substances. A notre connaissance, l'étude détaillée d'un cas simple comme celui que nous décrivons, reste à faire. Sans préjuger des résultats que l'on peut obtenir, on peut remarquer que la conductivité électrique conditionne l'existence de courants induits qui freinent les déplacements irréversibles de la paroi et allongent la durée des impulsions. Dans un isolant, on peut s'attendre à des impulsions de tension bien plus brèves et à des spectres de bruit s'étendant jusqu'à des MHz. D'autre part, les courants induits représentent une cause supplémentaire de dissipation d'énergie, mais la séparation des pertes par hystérésis et des pertes par courants induits est difficile, tant sur le plan des principes que du point de vue expérimental.

Nous avons également négligé l'aspect dynamique de la paroi. Dans ce qui suit il s'agit de déplacements à très faible vitesse.
L'effet de l'énergie d'échange est d'interdire à l'échelle microscopique toute variation brutale de l'orientation des moments.

1.2.2 L'énergie magnétostatique. - C'est l'énergie de couplage qui résulte des interactions coulombiennes entre moments magnétiques. Chaque moment élémentaire est placé dans le champ local dû à tous ses voisins. Il en résulte une énergie magnétostatique $U_{\mathrm{m}}$ de la forme :

$$
U_{\mathrm{m}}=-\frac{1}{2} \int \mathbf{J} . \mathbf{H}^{\prime} \mathrm{d} v
$$

$\mathbf{J}$ et $\mathbf{H}^{\prime}$ étant respectivement les vecteurs intensité d'aimantation et champ d'excitation au point considéré. L'intégrale est étendue à toute la matière aimantée.

Comme $\mathbf{H}^{\prime}$ et $\mathbf{B}^{\prime}=\mu_{0} \mathbf{H}^{\prime}+\mathbf{J}$ sont deux vecteurs continus différenciables, l'un irrotationnel, l'autre solénoïdal, nuls à l'infini; on démontre que l'intégrale étendue à tout l'espace

$$
\int \mathbf{H}^{\prime} \cdot \mathbf{B}^{\prime} \mathrm{d} v=0 .
$$

Il en résulte une autre expression de $U_{\mathrm{m}}$ :

$$
U_{\mathrm{m}}=\int \mu_{0} \mathbf{H}^{2} \mathrm{~d} v,
$$

l'intégrale étant étendue à tout l'espace.

Pour diminuer $U_{\mathrm{m}}$, il faut diminuer les masses magnétiques qui sont les sources de $\mathbf{H}^{\prime}$ et pour des sources données, limiter l'étendue du champ $\mathbf{H}^{\prime}$. D'un point de vue pratique, on abaissera l'énergie magnétostatique en éliminant les masses magnétiques ou en modifiant la répartition; par exemple en les regroupant en masses de signe opposé.

1.2.3 L'énergie d'anisotropie magnétocristalline. L'énergie interne du cristal saturé dépend de la direction commune des moments élémentaires par rapport aux axes du cristal. Il existe des directions cristallographiques pour lesquelles il faut dépenser moins d'énergie que pour d'autres pour aimanter le cristal à saturation. Ces directions sont dites de facile aimantation. La différence d'énergie nécessaire pour aimanter le cristal suivant une direction difficile et une direction facile mesure l'énergie d'anisotropie magnétocristalline. La figure 1 décrit la situation dans le fer, le nickel et le cobalt.

1.2.4 L'énergie magnétoélastique. - L'énergie magnétoélastique résulte de l'interaction entre la direction de l'aimantation et les déformations du réseau cristallin. Comme l'énergie d'anisotropie magnétocristalline dépend des déformations du réseau cristallin, celui-ci se déforme spontanément pour minimiser son énergie libre.

Un échantillon ferromagnétique de forme sphérique, à une température supérieure à celle de Curie, devient un ellipsoïde quand on le sature au-dessous de $T_{\mathrm{c}}$. 
$J(G)$
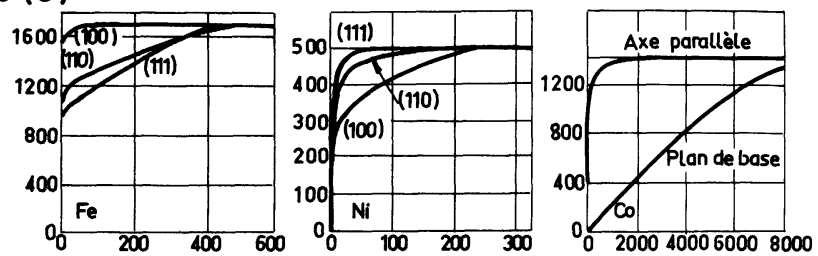

$H(O e)$

Fig. 1. - Courbes d'aimantation pour des monocristaux de fer, de nickel et de cobalt. Pour le fer, les courbes montrent que les directions $\langle 100\rangle$ sont des directions de facile aimantation et $\langle 111\rangle$ sont des directions de difficile aimantation. L'aimantation $M$ est exprimée en gauss alors que le champ $H$ l'est en orsted.

[Magnetization curves for single crystals of iron, nickel and cobalt. For iron, the curves show that the $\langle 100\rangle$ axes are directions of easy magnetization, and the $\langle 111\rangle$ axes are hard directions. The units for the magnetization $M$ and the field $H$ are the gauss and the œrsted respectively.]

Le couplage magnétoélastique se manifeste par la magnétostriction. Inversement, le fait de contraindre et de déformer - un matériau ferromagnétique peut modifier la direction des moments élémentaires.

Ce sont là les principales énergies responsables de l'apparition des domaines magnétiques dans un cristal parfait.

1.3 L'EXISTENCE DES DOMAINES. - L'échantillon se subdivise spontanément en domaines, c'est-à-dire en régions où l'aimantation est uniforme à une échelle intermédiaire entre l'échelle atomique et l'échelle de l'échantillon, afin d'abaisser son énergie interne.

Considérons l'exemple simple de la figure 2 . Ce cas correspond à celui d'une plaquette d'un matériau monocristallin du type fer taillé dans un plan de base

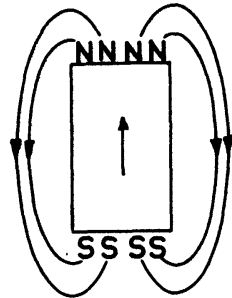

a

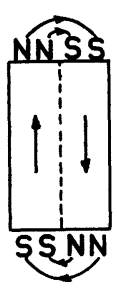

b

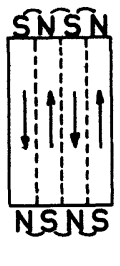

c

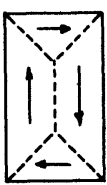

d

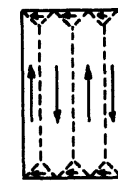

e
Fig. 2. - Origine des domaines.

[The origin of domains.]

du cube par exemple (100); les côtés étant parallèles aux axes de facile aimantation qui sont ici les axes quaternaires $\langle 010\rangle$ et $\langle 100\rangle$. La structure monodomaine $a$ conduit à une énergie interne élevée à cause de l'existence de l'énergie magnétostatique. De ce point de vue, $b$ et $c$ sont plus favorables et divisent approximativement cette dernière par 2 et 4 . $d$ et $e$ font disparaître les masses magnétiques restantes en fermant le flux du vecteur aimantation.
Un examen plus approfondi du problème montre que dans ce cas simple, on doit observer une structure du type $d$ ou $e$. L'expérience confirme d'ailleurs cette prévision. La structure en domaines résulte d'un compromis entre divers types d'énergie dont les effets sont contradictoires.

1.4 LeS PAROIS. - Il ne peut être question à l'échelle atomique de passer brutalement d'une direction d'aimantation à une autre car une telle façon de procéder demanderait une contribution beaucoup trop importante à l'énergie d'échange. Il existe donc entre deux domaines où les directions de l'aimantation sont différentes une zone de transition s'étendant sur un certain nombre de plans atomiques où l'aimantation tourne progressivement. C'est cette zone de transition qu'on appelle la paroi. Son épaisseur est liée au nombre de distances interatomiques sur laquelle se fait la transition. Sa structure est gouvernée par une compétition entre l'énergie d'échange et l'énergie d'anisotropie. La première a tendance à élargir la paroi, la seconde à la rétrécir. Il n'apparaît aucune énergie magnétostatique à l'intérieur de la substance tant que la composante de l'aimantation normale à la paroi se conserve. $\mathrm{Ce}$ fait très restrictif privilégie certaines orientations de parois. Une telle structure est caractérisée par une énergie interne qui lui est propre et qu'il est commode de ramener à l'unité de surface. La figure 3 montre la structure schématique d'une paroi de Bloch à $180^{\circ}$. Une telle paroi sépare deux domaines dont les vecteurs aimantation sont antiparallèles. Il existe également des parois de Bloch à $90^{\circ}$; elles séparent deux domaines dont les vecteurs aimantations sont perpendiculaires. Nous avons rencontré ce type de paroi dans les domaines de fermeture de la figure 2. Il existe de nombreux autres types de paroi qui peuvent se manifester soit parce que les conditions d'observation les favorisent, soit parce que les caractéristiques du matériau imposent une structure particulière.

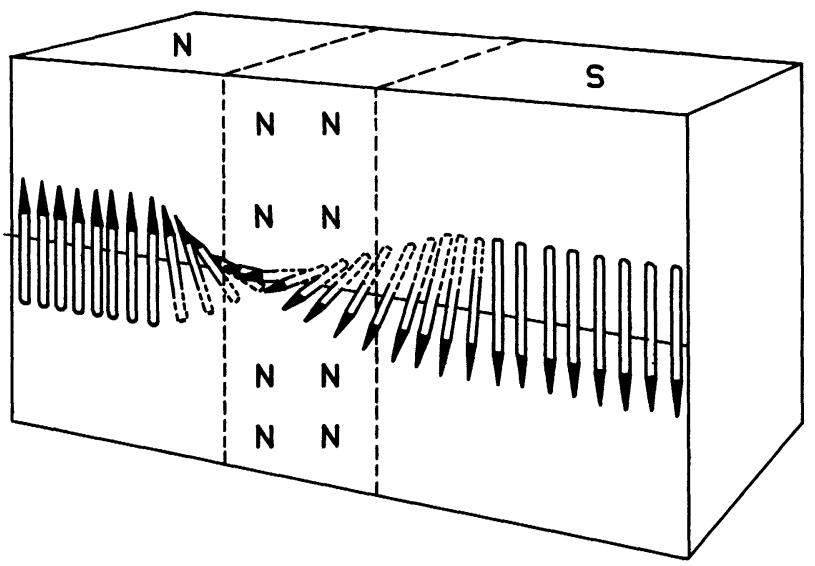

Fig. 3. - Structure de la paroi de Bloch séparant deux domaines. Dans le fer, l'épaisseur de la zone de transition est d'environ 300 paramètres de la maille.

[The structure of a Bloch wall between two domains. In iron, the thickness of the transition region is about 300 lattice constants.] 
1.5 LES CONFIGURATIONS EN DOMAINES ET LES PROCESSUS D'AIMANTATION. - 1.5.1 Il faut d'abord insister sur le fait que, sauf dans des cas très particuliers, on ne sait pas prévoir a priori, comment la matière va se subdiviser en domaines. Le problème est très sérieusement compliqué par l'existence des conditions aux limites qui sont imposées par la surface du cristal. La détermination de la configuration d'énergie minimale est un problème difficile et complexe que l'on ne sait pas résoudre en général. Même si on savait le résoudre, on n'observerait pas pour autant la structure correspondante. En effet les configurations réelles ne réalisent pas des minima d'énergie absolue. Ce fait se traduit par le choix d'une des configurations en domaines distinctes mais d'énergies très voisines, correspondant à des conditions extérieures bien déterminées. C'est ce choix qui est à l'origine des phénomènes d'hystérésis.

1.5.2 Les processus d'aimantation. - L'application d'un champ magnétique extérieur ne peut provoquer que la rotation des moments élémentaires. Cette rotation est plus facile dans les parois et dans leur voisinage immédiat : c'est là qu'elle se produira dans les champs faibles. Tout se passe comme si la paroi s'était déplacée et reformée un peu plus loin. Ces déplacements de parois font que certains domaines convenablement orientés par rapport au champ extérieur appliqué voient leur volume croître au détriment des autres. Ce processus fait disparaître progressivement le système de parois quand on fait croître le champ magnétisant.

Dans une deuxième phase, les moments vont s'écarter en bloc de leur position et tourner progressivement de manière à s'aligner suivant le champ appliqué. C'est le domaine des rotations. Elles ont un caractère réversible et se produisent dans des champs plus élevés. Elles sont d'autant plus faciles que l'énergie d'anisotropie magnétocristalline est plus petite. On peut ainsi grossièrement attribuer aux diverses régions de la courbe d'aimantation des processus d'aimantation prépondérants. Nous allons montrer sur un exemple concret que la réalité est plus complexe. La figure $4 \mathrm{~A}$ représente le cas théorique que nous avions envisagé figure $2 d$. Quand on applique un champ extérieur parallèle au grand côté, on doit voir le domaine où l'aimantation est suivant le champ, croître, et les domaines de fermeture se modifier.

La figure 4, B, C, D, E, F, G montre l'évolution des domaines quand, après avoir fait croître le champ, on le ramène à zéro. Nous avons ici un très bel exemple de ce qu'est l'hystérésis.

Quand après avoir appliqué un champ suffisant pour faire disparaître les domaines et aligner les moments, on fait décroître celui-ci, les domaines ne réapparaissent pas tout de suite. Il apparaît d'abord des germes d'aimantation inverse à partir desquels se développe la nouvelle structure en domaines. C'est le phénomène de nucléation. Le champ moyen de nucléation des domaines inverses est le champ
A

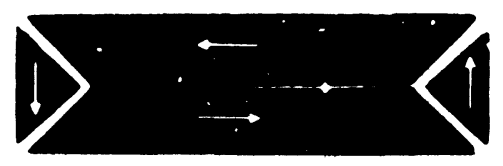

B

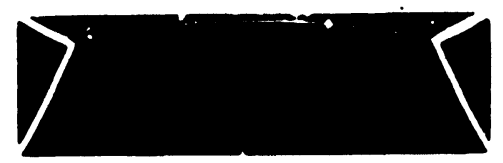

C

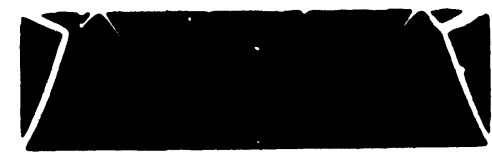

D

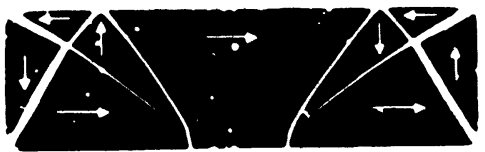

E

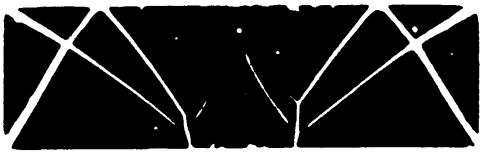

$\mathbf{F}$

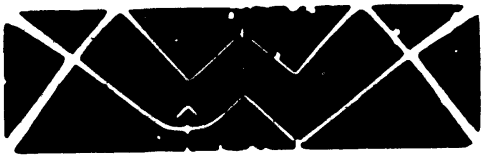

$\mathbf{G}$

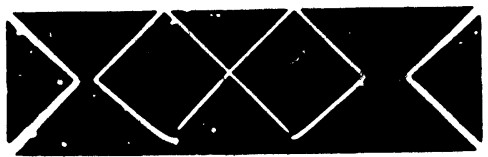

Fig. 4. - Etude de domaines sous champ magnétique appliqué parallèlement au grand côté d'une plaquette rectangulaire de $\mathrm{Ni}-\mathrm{Co}$. Il s'agit d'un cristal pratiquement parfait, de type whisker et de dimensions : $112 \times 35 \times 0,55(\mu . \mathrm{m})^{3}$. A) $H=0$, configuration d'équilibre. B) $H=5,1 \mathrm{Oe}$, noter la courbure de la paroi à $180^{\circ}$ ancrée à ses 2 extrémités par les domaines de fermeture. C) $H=6,0 \mathrm{Oe}$, la paroi à $180^{\circ}$ disparaît. D) $H=1,8 \mathrm{Oe}$, la configuration initiale ne réapparaît pas spontanément quand le champ décroît. E) et F) $H=1,4 \mathrm{Oe}$, on observe une modification spontanée dans l'arrangement des domaines. G) $H=0$, on retrouve une configuration métastable. D'après R. W. de Blois, G.E. Laboratory Rep. No 65-C-082 (Déc. 1965).

[The behaviour of a domain pattern in a magnetic field applied parallel to the length of a rectangular $\mathrm{Ni}-\mathrm{Co}$ platelet. The sample is an almost perfect single crystal of the whisker type and its dimensions are : $112 \times 35 \times 0.55(\mu \mathrm{m})^{3}$. A) $H=0$, equilibrium configuration. B) $H=5.1 \mathrm{Oe}$, note the vaulting of the $180^{\circ}$ wall anchored at both ends by the closure domains. C) $H=6.0 \mathrm{Oe}$, the $180^{\circ}$ wall disappears. D) $H=1.8 \mathrm{Oe}$, the initial configuration does not reappear spontaneously when the field decreases. E) and F) $H=1.4 \mathrm{Oe}$, a spontaneous change is observed in the domain topology. G) $H=0$, the system returns to a metastable configuration. From R. W. de Blois, G.E. Laboratory Rep. No 65-C-082 (Dec. 1965).]

coercitif de la substance. On peut également envisager celui-ci comme le champ seuil nécessaire pour que les parois balayent les domaines. 
2. Cas d'une paroi de Bloch à $180^{\circ}$ unique se déplaçant dans un milieu aléatoirement perturbé. -2.1 STRUCTURE EN DOMAINES D'UN CADRE MONOCRISTALLIN AYANT SES CÔTÉS PARALlÈles AUX AXES DE FACILE AIMANTATION. - Un cadre monocristallin d'une substance du type fer taillé comme l'indique la figure 5 a une structure en domaine très simple, caractérisée par une seule paroi de Bloch à $180^{\circ}$ mobile et quatre parois à $90^{\circ}$ fixes assurant la fermeture du flux.


$b^{\prime}=10,50 \mathrm{~mm}$

époisseur $=0,61 \mathrm{~mm}$

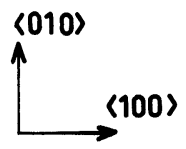

Fig. 5. - Dimensions et structure en domaines du cadre monocristallin de fer-silicium. La surface moyenne de la paroi est $0,291 \mathrm{~cm}^{2}$.

[Dimensions and domain structure of the single-crystal $\mathrm{Fe}-\mathrm{Si}$ frame. The average area of the $180^{\circ}$ wall is $0.291 \mathrm{~cm}^{2}$.]

L'application d'un champ magnétique, créé par un enroulement solénoïdal disposé sur les bras du cadre, permet de déplacer la paroi. Quand on fait croître le champ, le domaine dont l'aimantation est suivant celui-ci voit son volume augmenter et occuper progressivement tout le volume du cadre. Avec un tel échantillon, il est également possible d'obtenir une structure en domaines comportant $0,1,2,3$ parois de Bloch à $180^{\circ}$. Remarquons que la structure d'énergie minimale est celle où il n'y a pas de paroi à $180^{\circ}$, on ne l'obtient cependant que très rarement.

2.2 L'INFLUENCE DES DÉFAUTS. - Tout ce qui précède a été dit pour un cristal parfait. Or les cristaux que l'on sait réaliser ne sont jamais parfaits et comportent toujours des défauts : dislocations, joints de grains, trous, lacunes, inclusions... On peut souvent considérer que ces défauts sont aléatoirement repartis. Du fait de l'existence des défauts, la densité d'énergie superficielle de la paroi varie en fonction de sa position dans le cristal. Pour traiter rigoureusement le problème, il faudrait connaître la répartition des défauts en chaque point et savoir calculer leur interaction avec la paroi. On ne sait pas faire un tel calcul, et on est conduit à envisager des modèles $\left(^{2}\right)$. L'un de ceuxci consiste à considérer l'énergie superficielle de la

$\left({ }^{2}\right)$ Remarque : Dans ce qui suit nous traiterons des modèles de fonction potentiel. Ce sont les plus élaborés et ceux qui ont été le plus étudiés. Ils présentent la propriété de conservativité (cf. 3.2; 2.2). Nous donnons en annexe un exemple de modèle non conservatif. paroi comme une fonction aléatoire de sa position. Dans le cas précédent, avec une paroi plane en moyenne, le problème est à une dimension. On posera :

$$
V(x)=V+\tilde{V}(x) .
$$

La partie principale $V$ de l'énergie superficielle de la paroi $V(x)$ est la grandeur déterministe très voisine de celle qui résulterait de la théorie du cristal parfait. Elle est indépendante de $x$ et vaut $E\{V(x)\}$ où $E$ symbolise l'espérance mathématique. $\tilde{V}(x)$ est la partie aléatoire de $V(x)$.

2.3 Mouvement de la paroi sous L'aCtion d'Un CHAMP MAGNÉTIQUE. COURBE DE PREMIÈRe AIMANTATION. CYCLE D'HYSTÉRÉSIS ÉLÉMENTAIRE, CHAMP COERCIIIF. - Soit une paroi de Bloch séparant deux domaines d'aimantation $\mathbf{J}_{1}$ et $\mathbf{J}_{2}$. Appliquons un champ magnétique interne $\mathbf{H}$; sous l'influence du champ, la paroi se déplace. La variation d'énergie interne de la paroi s'écrit :

$$
\mathrm{d} V_{\mathrm{p}}=\mathrm{d} V / \mathrm{d} x S \mathrm{~d} x
$$

$S$ étant la surface de la paroi ; alors que la variation d'énergie potentielle due à la présence du champ $\mathbf{H}$ vaut :

$$
\mathrm{d} V_{H}=-\left(\mathbf{J}_{2}-\mathbf{J}_{1}\right) \mathbf{H} S \mathrm{~d} x .
$$

La paroi s'arrêtera quand $\left(\mathbf{J}_{2}-\mathbf{J}_{1}\right) \mathbf{H}=\mathrm{d} V / \mathrm{d} x$. La quantité $\left(\mathbf{J}_{2}-\mathbf{J}_{1}\right) \mathbf{H}$ est analogue à un terme de pression hydrostatique; on l'appelle parfois la pression magnétique. L'équilibre sera stable $\operatorname{si~}^{2} V / \mathrm{d} x^{2}>0$. Dans le cas particulier de la paroi de Bloch à $180^{\circ}$ : $\mathbf{J}_{2}=-\mathbf{J}_{1}=\mathbf{J}_{\mathrm{s}}$ et on a à l'équilibre $2 J_{\mathrm{s}} H=\mathrm{d} V / \mathrm{d} x$. Dans le modèle à une dimension précédent, l'étude des déplacements de la paroi se ramène à l'étude du mouvement d'un point $M$ sur la courbe $V(x)$ sous l'action du champ appliqué (Fig. 6). En champ nul, le point $M$ se trouve dans une position d'équilibre

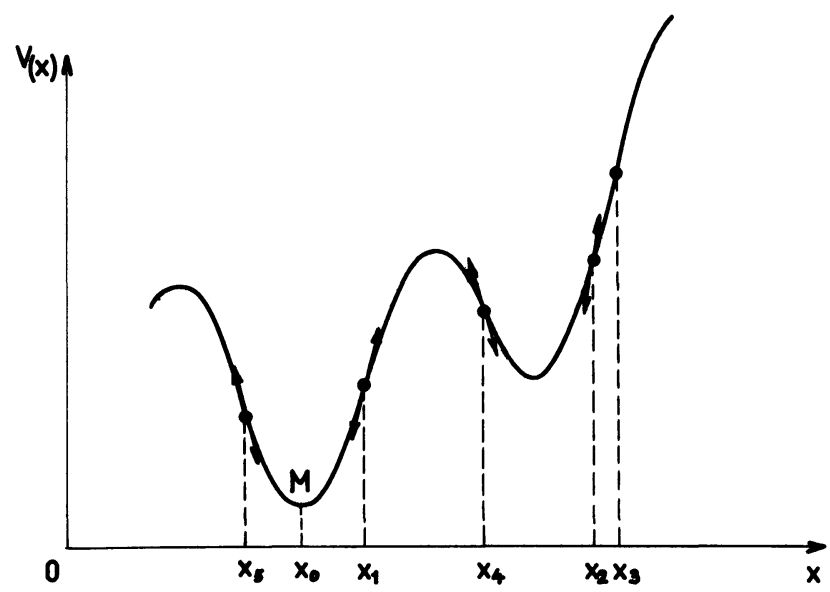

Fig. 6. - Déplacements réversibles et irréversibles d'une paroi dans un potentiel aléatoire $V(x)$.

[Reversible and irreversible motion of a wall in a random potential energy $V(x)$.] 
repérée par son abscisse $x_{0}$ correspondant à un minimum de $V(x)$. Lorsqu'on applique un champ magnétique croissant, $M$ commence à se déplacer réversiblement jusqu'au point où la pente $\mathrm{d} V / \mathrm{d} x$ équilibre la pression magnétique. Quand $\mathrm{M}$ atteint un point d'inflexion d'abscisse $x_{1}$, il saute irréversiblement jusqu'au premier point d'abscisse $x_{2}$ où la pente $\mathrm{d} V / \mathrm{d} x$ est suffisante pour arrêter la paroi. Si le champ varie de façon monotone, on observe ainsi une succession d'accroissements d'aimantation alternativement réversibles et irréversibles. Si au contraire, on inverse le sens de variation du champ lorsque la paroi a atteint la position $x_{3}$, on observe un déplacement réversible $x_{3} x_{4}$ suivi d'un saut irréversible $x_{4} x_{5}$ en sens inverse de $x_{2} x_{3}$. Ainsi apparaît un cycle d'hystérésis élémentaire (Fig. 7).

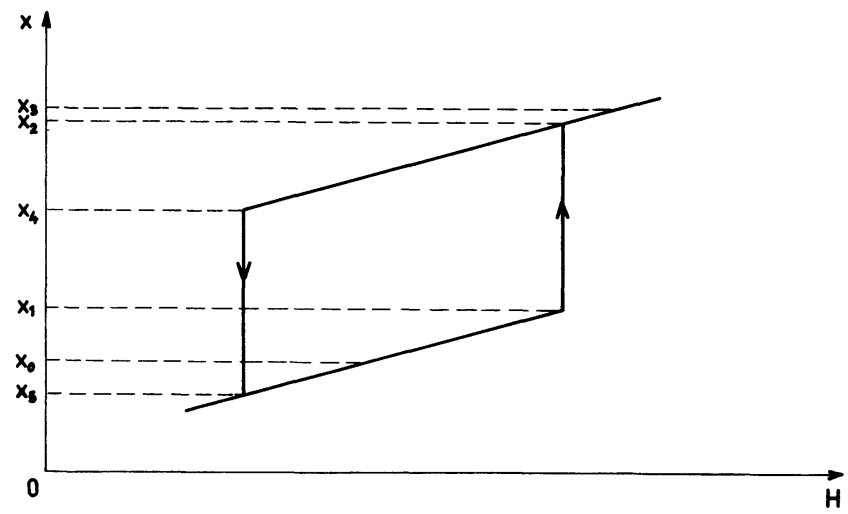

Fig. 7. - Cycle élémentaire d'une paroi dans un potentiel aléatoire : $x_{0} x_{1}, x_{2} x_{3}, x_{3} x_{4}$ : déplacements réversibles; $x_{1} x_{2}, x_{4} x_{5}$ : déplacements irréversibles.

[Elementary cycle of a wall in a random potential : $x_{0} x_{1}, x_{2} x_{3}$, $x_{3} x_{4}$ : reversible motion; $x_{1} x_{2}, x_{4} x_{5}$ : irreversible motion.]

Dans un tel modèle, le champ coercitif est lié à la plus grande pente que peut rencontrer la paroi, on a :

$$
H_{\mathrm{c}}=\frac{1}{2 J_{\mathrm{s}}}\left(\frac{\mathrm{d} V}{\mathrm{~d} x}\right)_{\max } .
$$

2.4 "SAUTS De BarkHAUSEN »; " BRUIT De BarKHAUSAN" (Fig. 8). - Lors d'un déplacement irréversible de la paroi du type $x_{1} x_{2}$ ou $x_{4} x_{5}$ (Fig. 7), l'aimantation varie brusquement de $\Delta J$. Il en résulte une variation de flux d'induction à travers un bobinage placé sur le cadre (Fig. 5) et une f.e.m. aux bornes de celui-ci.

Comme les moments mettent un temps non nul pour se retourner et que dès que le processus de retournement est amorcé, il se développe dans la substance, au voisinage des moments retournés, des courants de Foucault qui freinent leur retournement, la variation de flux s'étale sur un certain temps. La f.e.m. induite se présente comme une impulsion à front raide, suivie d'une décroissance exponentielle. La forme de l'impulsion est essentiellement déterminée, dans les substances conductrices, par les courants induits.
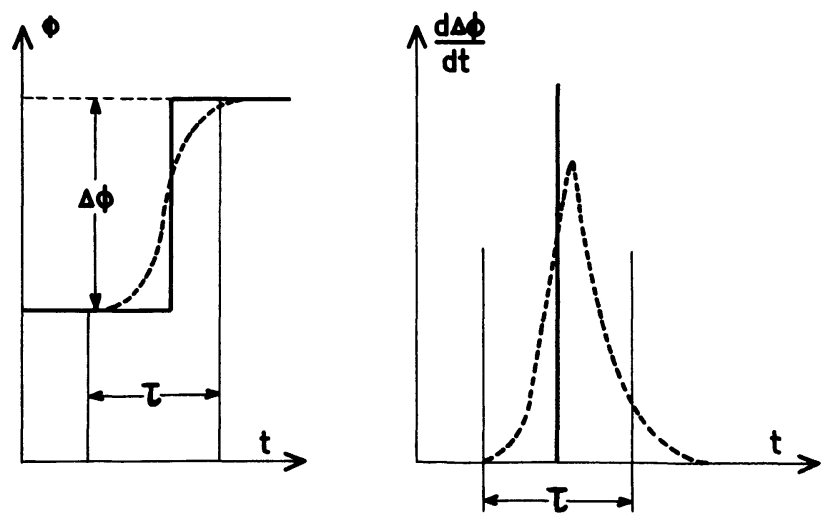

Fig. 8. - Variation d'induction et f.e.m. induite par un saut de Barkhausen.

[Change of induction and voltage induced by a Barkhausen jump.]

On a coutume de nommer saut de Barkhausen une impulsion de ce type et bruit de Barkausen la succession de ces impulsions lors d'un processus d'aimantation par déplacement de paroi.

2.5 ACtion de L'AGitation thermique. - Du fait de l'existence de l'agitation thermique, les moments élémentaires fluctuent très rapidement au cours du temps autour de leur valeur moyenne. Il en résulte dans la substance un champ magnétique interne aléatoire fluctuant autour d'une valeur moyenne nulle à des fréquences très élevées.

L'action de ce champ peut s'ajouter à celle du champ appliqué et rendre possible le passage de barrières de potentiel. $\mathrm{Si}$, par exemple, on amène la paroi au voisinage de $x_{1}$ en appliquant un champ magnétique convenable et qu'on maintienne le champ pendant un certain temps, on peut observer le saut de $x_{1}$ en $x_{2}$ (cf. Fig. 6).

Du fait de l'existence de l'agitation thermique, il s'introduit un autre caractère aléatoire fondamental dans les processus d'aimantation.

2.6 LeS MODÈLES DE LA FONCTION POTENTIEL $V(x)$. - Le modèle de fonction potentiel a été introduit par L. Néel en 1942 [3] dans un mémoire consacré à l'étude des lois de Rayleigh. Il propose pour $V(x)$ un contour polygonal caractérisé : par une subdivision de l'axe des $x$ en intervalles disjoints et adjacents, de longueur $2 l$ constante, par un tirage au sort de la pente de chaque segment suivant une loi gaussienne centrée de variance $l / 2 P_{0}^{2}$ et par une absence de corrélation entre les pentes des segments successifs. Pour éviter une discontinuité de $\mathrm{d} V / \mathrm{d} x$ qui n'est pas dans la nature physique du problème, Néel remplace les segments de droite par des arcs de parabole. $V(x)$ est alors entièrement défini par $l$ et $P_{0}$. Le problème consiste à étudier quantitativement le mouvement du point $\mathrm{M}$ représentatif de la paroi sur la courbe $V(x)$ (cf. 2.3).

Pour cela définissons : le champ réduit 
$h=\mathbf{H}\left(\mathbf{J}_{2}-\mathbf{J}_{1}\right) / P_{0}$, la valeur $\varepsilon$ de ce champ correspondant au champ maximum $H_{\mathrm{m}}$ appliqué et la pente réduite $p=P / P_{0}$. Nous supposerons que

$$
|h|<\varepsilon \ll 1,
$$

ce qui revient physiquement à dire que le champ maximal appliqué reste petit devant le champ coercitif. On définit alors trois catégories A, B, C, de côtés du contour polygonal dont on situe les pentes $p$ par rapport à $\varepsilon$ :

$$
\mathrm{A}: p>\varepsilon ; \quad \mathrm{B}:|p|<\varepsilon ; \quad \mathrm{C}: p<-\varepsilon .
$$

Néel montre alors que l'on peut décomposer la courbe $V(x)$ de façon biunivoque en domaines qui appartiennent aux quatre types suivants : $\mathrm{AB}^{n} \mathrm{~A}, \mathrm{AB}^{n} \mathrm{C}$ $\mathrm{CB}^{n} \mathrm{~A}$ et $\mathrm{CB}^{n} \mathrm{C}$, où $n$ est en entier positif ou nul. $\mathrm{AB}^{n} \mathrm{C}$ représente une portion de contour polygonal constitué par une suite de $n+2$ côtés dont le premier est du type A, les $n$ suivants du type B et le dernier du type $C$. L'intérêt de cette subdivision en domaines réside dans les deux faits suivants : Après une désaimantation par un champ alternatif lentement décroissant la paroi se trouve obligatoirement dans un domaine $\mathrm{CB}^{n} \mathrm{~A}$; lors de l'application ultérieure d'un champ réduit $h$ dont le module n'excède pas $\varepsilon$, la paroi reste à l'intérieur du domaine $\mathrm{CB}^{n} \mathrm{~A}$ qui la contient initialement. Dès lors le problème se ramène à l'étude statistique des domaines $\mathrm{CB}^{n} \mathrm{~A}$ en fonction de $\varepsilon$ à savoir : leur nombre, leur longueur (valeur de $n$ ) et leur probabilité d'avoir capté une paroi au cours de la désaimantation. On en déduit l'espérance mathématique du déplacement de la paroi et donc la courbe d'aimantation. En prenant $h$ et $\varepsilon$ du même ordre de grandeur (tous les deux petits devant l'unité) et en négligeant dans l'aimantation totale les termes du troisième ordre, on montre qu il suffit de tenir compte des domaines $\mathrm{CA}, \mathrm{CBA}$ et $\mathrm{CB}^{2} \mathrm{~A}$. L'aimantation se réduit à deux termes, l'un réversible et proportionnel à $h$, l'autre irréversible et proportionnel à $h^{2}$. On a :

$$
i=\lambda h+\frac{h^{2}}{\pi}
$$

où $i=x / 2 l$ est l'aimantation réduite, pour la courbe de première aimantation et

$$
i=\lambda h \pm \frac{h^{2}}{2 \pi}
$$

pour les branches ascendantes et descendantes du cycle d'hystérésis. L'importance du résultat obtenu tient moins à la valeur des coefficients trouvés qu'à la nature remarquable du développement en série des puissances croissantes de $h$ obtenu, dont les termes impairs sont réversibles alors que les termes pairs sont irréversibles. Ce résultat reste valable quelle que soit la loi de probabilité régissant la répartition des pentes du contour polygonal. Il faut toutefois noter l'hypothèse physique essentielle de ce modèle : une seule variable d'espace suffit à décrire les déplacements de la paroi (paroi non flexible).

Néel étend ensuite le calcul au cas d'un polycristal où les mécanismes d'aimantation se bornent aux déplacements de parois : il traite le cas d'un polycristal d'une substance magnétiquement uniaxiale et esquisse celui d'un matériau comme le fer, dont l'anisotropie magnétique est de symétrie cubique. On obtient une loi macroscopique analogue à celle obtenue pour la paroi de Bloch isolée et du type $J=a H+b H^{2}$ pour la courbe de première aimantation. Le premier terme caractérise l'aimantation réversible, le second l'aimantation irréversible. Les cycles d'hystérésis sont également paraboliques $\left({ }^{3}\right)$. Des lois analogues avaient été trouvées par Lord Rayleigh en 1887 [4] pour le comportement des polycristaux en champs faibles.

Ce modèle a été repris par G. Bonnet (1967) [6] qui, à partir de la théorie des fonctions aléatoires, l'a justifié et notablement complété dans le cas de perturbations à petite échelle dont la distribution est homogène dans toute la substance. Il trouve alors pour $V(x)$ une fonction en dent de scie analogue à celle introduite par Néel. Nous reviendrons ultérieurement sur ce travail.

3. Etude expérimentale du déplacement d'une seule paroi de Bloch à $180^{\circ}$ dans un milieu aléatoirement perturbé. - Nous avons utilisé pour cette étude un cadre monocristallin de $\mathrm{Fe}-\mathrm{Si}$ contenant environ $3 \%$ de silicium en poids. L'étude topographique aux rayons $\mathrm{X}$ montre que l'essentiel des défauts est constitué par un réseau de dislocations isolées, réparties au hasard dont la densité est de l'ordre de $10^{4} \mathrm{~cm}^{-2}$. L'échelle des défauts est donc de quelques dizaines de microns. A cela s'ajoutent des sous-joints de grain très peu désorientés; leur échelle est de l'ordre de quelques millimètres.

Nous avons essentiellement développé quatre points au cours de cette étude. Nous nous sommes d'abord demandé si la paroi se comportait comme un objet déformable lors de l'application d'un champ magnétique ou sous l'influence de l'agitation thermique. Nous voulions également tester la validité du modèle de la fonction potentiel et en préciser les limites. Nous avons ensuite envisagé une façon plus générale de décrire le déplacement de la paroi à partir de la force exercée sur celle-ci par les défauts du réseau. Ce dernier point nous a permis de mettre en évidence d'autres aspects de son comportement.

$\left({ }^{3}\right)$ Remarque : L'énergie dissipée par hystérésis est $\sim H^{3}$, puisque l'aire du cycle d'hystérésis est $4 / 3 b H_{\max }^{3}$. Remarquons que dans un système non linéaire hors d'équilibre une dissipation d'énergie de ce type caractérise une faible métastabilité. Si l'échantillon est désaimanté thermiquement, c'est-à-dire porté au-dessus de la température de Curie et refroidi en champ nul, la structure en domaine obtenue est hautement métastable. Lors de la première application du champ, la substance ne suit pas la loi de Rayleigh. Cette remarque permet de considérer les faits décrits au paragraphe 3.2.1 et 3 , d'un point de vue plus général. 
Un dernier paragraphe a été consacré à l'étude de l'influence des fluctuations thermiques sur le déplacement de la paroi. Il nous a permis de préciser, d'une part le rôle des fluctuations thermiques et de certaines grandeurs qui leur sont attachées; d'autre part, de préciser quelques caractères de la paroi (flexibilité) et des impuretés du cristal (obstacles).

Il peut paraître surprenant que de telles vérifications directes n'aient pas déjà été faites. Mais pour pouvoir les mener à bien, il faut disposer à la fois de monocristaux de grande taille et d'excellente qualité et d'un appareillage de mesure très performant.

3.1 LE DISPOSITIF EXPÉRIMENTAL; SES POSSIBILITÉs. - Divers enroulements répartis sur les côtés du cadre permettent de le désaimanter par un champ alternatif lentement décroissant, d'appliquer le champ magnétisant et de recueillir les variations de flux proportionnelles aux déplacements de la paroi.

Dans toutes ces expériences, nous avons utilisé un fluxmètre galvanométrique, particulièrement sensible et stable [6]. Nous l'avons utilisé soit tel quel, soit en fluxmètre asservi. En effet, lorsqu'on applique un champ magnétique à variation monotone, la taille des sauts croît en moyenne (Fig. 9) $\left({ }^{4}\right)$ et la paroi franchit irréversiblement un nombre croissant de maxima de $V(x)$; toute l'information contenue dans ces déplacements est perdue. Pour tourner cette difficulté,

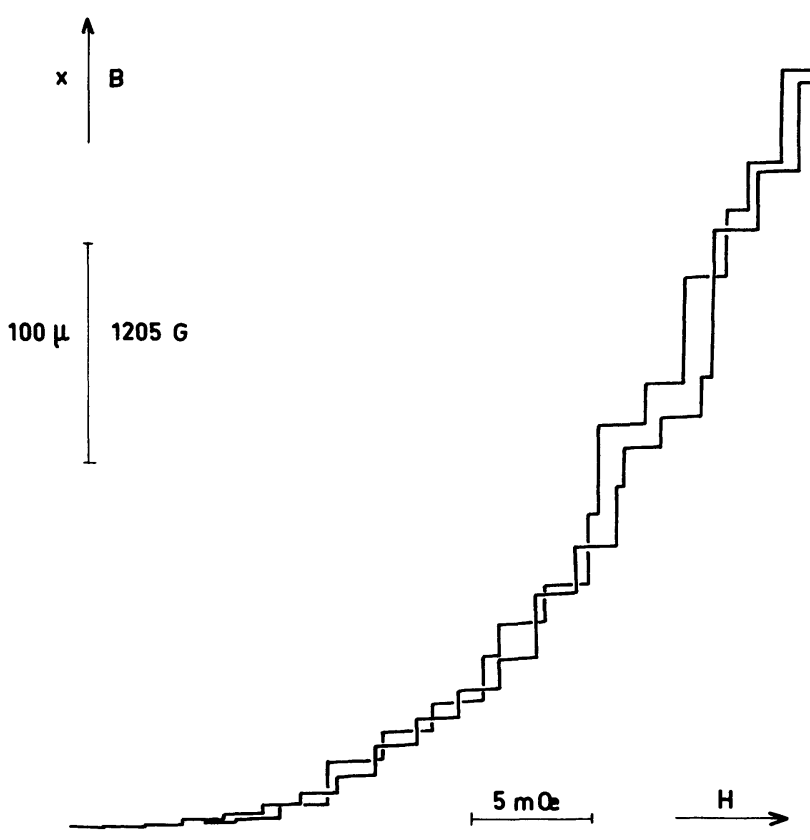

Fig. 9. - Deux exemples de courbe de première aimantation dans des champs relativement faibles $\left(\sim H_{\mathrm{c}}\right)$.

[Two examples of the virgin curve $\left(H \sim H_{\mathrm{c}}\right)$.]

$\left({ }^{4}\right)$ Remarque : De nombreuses figures : 9, 13, 14, 16, 20, 21, 22 , $24,26,28,36$, correspondent à des enregistrements obtenus sur une table traçante $x, y$ ou $y(t)$. Nous connaissons les échelles sur les axes, mais nous ignorons en général l'origine. C'est pour cela que nous avons adopté cette présentation. nous avons utilisé une technique déjà décrite [7] qui consiste à asservir les déplacements de la paroi (Fig. 10). L'échantillon est inclus dans une boucle de retour qui compare la tension induite $-\frac{\mathrm{d} \varphi}{\mathrm{d} t}$ à une tension de consigne $e_{\mathrm{c}}$. Si la paroi est rigide, on lui impose ainsi de se déplacer à une vitesse constante.

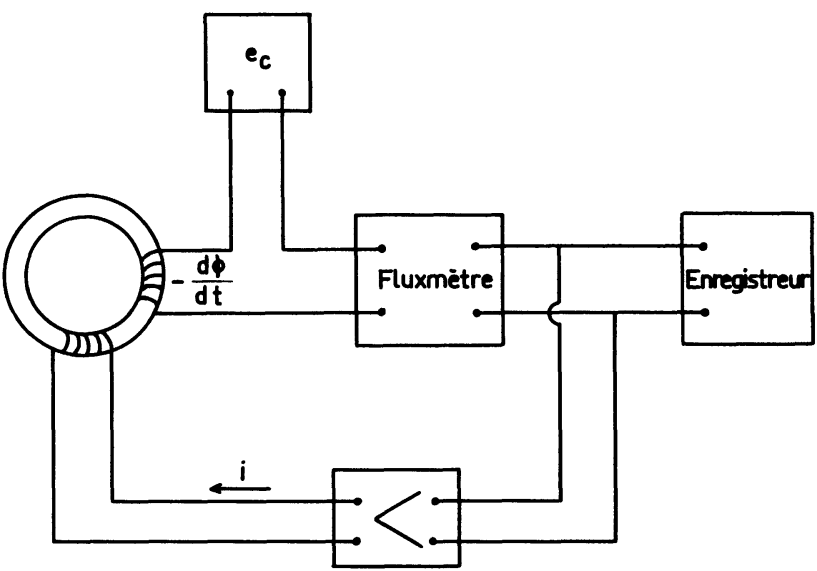

Fig. 10. - Contrôle des déplacements de la paroi par une boucle d'asservissement.

[Controlling the motion of the wall by a feedback loop.]

Lorsqu'un saut irréversible se déclenche, l'asservissement réagit et applique aussitôt une variation négative de champ qui freine le déplacement de la paroi. Avec un taux d'asservissement convenable, on impose à la paroi de décrire la fonction potentiel en sautant les barrières d'énergie une par une. Une chaîne amplificatrice constituée essentiellement d'un deuxième amplificateur galvanométrique, permet d'enregistrer la tension induite dans un enroulement de mesure, c'est-àdire le bruit de Barkhausen dû au déplacement de la paroi à $180^{\circ}$. Celui-ci peut également être obtenu à partir de la chaîne servant à l'asservissement. L'étude statistique des séquences de sauts et la partie traitement du signal ont été effectuées au CEN-G (LETI, MCTE Division Traitement du Signal).

3.2 LES RÉSULTATS EXPÉRIMENTAUX. - 3.2.1 La courbe de première aimantation, la loi de Rayleigh élémentaire. - Lorsque, après avoir désaimanté l'échantillon, on lui applique un champ magnétique pas trop élevé vis-à-vis du champ coercitif $\left(H_{\mathrm{c}}=28 \mathrm{mOe}\right)$, on obtient des enregistrements semblables à ceux de la figure 9 . On observe bien une succession de déplacements réversibles et irréversibles. Etant donné leur faible amplitude, les déplacements réversibles apparaissent à cette échelle comme des palliers pratiquement horizontaux. On constate de plus que la taille des sauts croît en moyenne avec le champ magnétique $H$. Cela est en accord avec le modèle de la fonction potentiel : si l'on admet que les valeurs de la pente $P=\mathrm{d} v / \mathrm{d} x$ aux points d'inflexion sont distribuées suivant une certaine loi de probabilité, les 
chances de rencontrer une pente suffisante pour arrêter la paroi décroissent lorsque $H$ croît. Par conséquent, la paroi franchit, au cours de ses déplacements irréversibles, un nombre de plus en plus grand de maxima de la fonction potentiel.

Après une désaimantation alternative, la paroi peut occuper dans le cristal une position initiale à peu près quelconque. On a donc la possibilité d'étudier ses déplacements dans les champs faibles en divers points du cristal. Comme on pouvait s'y attendre, nous avons constaté que les successions de mouvements réversibles et irréversibles diffèrent les unes des autres suivant la position initiale de la paroi. Par contre, les courbes moyennes que l'on peut tracer sur ces diverses lois d'aimantation sont bien superposables les unes aux autres (Fig. 9) : la courbe de première aimantation est donc statistiquement bien définie et caractéristique du cristal. Elle est de plus d'allure parabolique en champs faibles.

Nous avons d'ailleurs enregistré un certain nombre de ces courbes et nous avons porté l'amplitude $\Delta x$ des sauts irréversibles en fonction du champ magnétique pour lequel ils se déclenchent (Fig. 11). On trouve un nuage de points, ce qui est tout à fait normal puisqu'il s'agit d'un phénomène aléatoire ; on constate de plus que les résultats de diverses expériences se répartissent apparemment au hasard dans le nuage de points. Cette remarque confirme que le monocristal étudié est homogène à une échelle suffisante pour qu'on puisse tirer des conclusions statistiques valables.

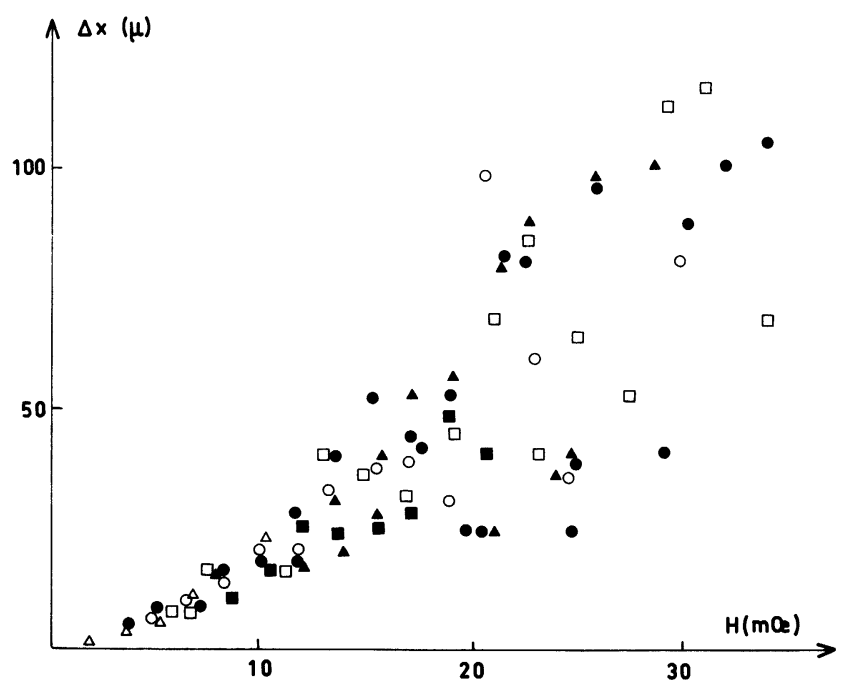

Fig. 11. - Croissance de la taille des sauts de Barkhausen en fonction du champ magnétique. La figure regroupe les résultats de plusieurs expériences.

[Dependence of the size of Barkhausen jumps on the magnetic field (results of several experiments).]

On constate que les points sont relativement bien groupés autour d'une droite dans les champs ne dépassant pas $20 \mathrm{mOe}$, soit environ $70 \%$ du champ coercitif. Cette décroissance linéaire de la taille moyenne des sauts montre que l'aimantation irréversible totale acquise par application d'un champ $H$ pas trop élevé est proportionnelle à $H^{2}$. On justifie ainsi très simplement le terme quadratique de la loi de Rayleigh. Lorsque le champ devient supérieur à 20 m@e environ, la taille moyenne des sauts croît plus vite qu'une loi linéaire.

3.2.2 La paroi se comporte comme un objet rigide; les arguments en faveur du modèle $V(x)$. - 3.2.2.1. La première question qui se pose est de savoir si les déplacements de la paroi à $180^{\circ}$ dans chaque bras du cadre sont ou non fortement corrélés quand on applique un champ magnétique. L'examen de la figure 9 montre que si l'on applique à la paroi un champ magnétique croissant avec le temps, le bruit de Barkhausen observé n'est pas stationnaire. Par contre, si on opère en fluxmètre asservi, on impose à la paroi de décrire un par un les accidents de la fonction potentiel et le bruit observé est alors stationnaire, à condition toutefois que le milieu aléatoirement perturbé soit homogène, condition qui est bien réalisée.

Nous avons enregistré dans ces conditions simultanément les deux séquences de sauts sur les deux bras opposés du cadre. Les deux chaînes de mesure étaient identiques et l'enroulement de champ magnétisant était bobiné sur un seul bras du cadre. Nous avons déterminé les fonctions d'autocorrélation de chaque séquence ainsi que la fonction d'intercorrélation des deux séquences. Nous avons ensuite comparé les autospectres à l'interspectre $\left({ }^{5}\right)$. La figure 12 montre que nous avons un très fort couplage. Nous pouvons en conclure qu'à la précision où sont faites les mesures, la paroi se comporte comme un objet rigide faisant le tour du cadre et se déplaçant en bloc.

3.2.2.2 Lors de deux ou plusieurs passages successifs la paroi a-t-elle le même comportement ? La propriété de conservativité est-elle vérifiée ? - La figure 13 reproduit deux enregistrements d'un même cycle d'hystérésis partiel décrit assez près de la saturation positive. On retrouve une alternance de déplacements réversibles et de sauts irréversibles. On remarque de plus une forte ressemblance entre les deux tracés successifs.

Nous avons également constaté que les cycles d'hystérésis décrits dans les champs faibles $(H<20 \mathrm{mOe})$ sont limités par des courbes moyennes paraboliques obéissant aux lois de Rayleigh. De plus, deux cycles successifs décrits dans les mêmes conditions sont parfaitement superposables.

Lorsque les champs magnétiques appliqués deviennent très faibles, on enregistre des cycles d'hystérésis

$\left({ }^{5}\right)$ Remarque : Le bruit étant constitué d'impulsions brèves, son spectre s'étend jusqu'à des fréquences de l'ordre de plusieurs kilohertz. En fait, nous l'avons filtré pour ne conserver que les basses fréquences caractérisant la succession des sauts. Les spectres de puissance ont été obtenus à l'aide d'un corrélateur numérique CTR 1024 et d'un transformateur de Fourier TFN 1024 Schlumberger. 


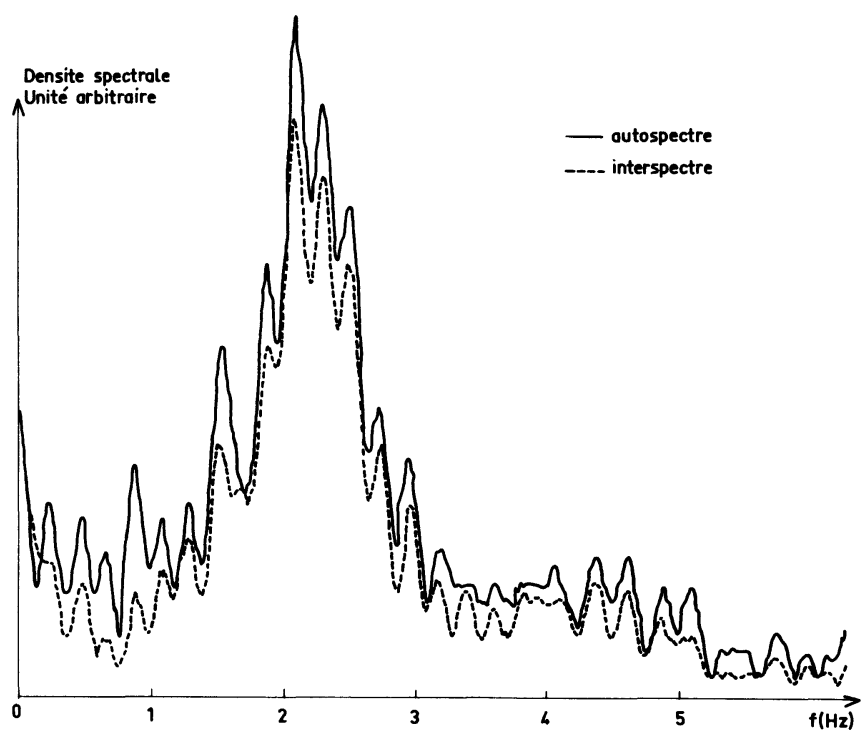

Fig. 12. - Densités spectrales énergétiques de deux séquences de sauts Barkhausen enregistrés simultanément sur les deux bras opposés du cadre. L'examen de l'autospectre et de l'interspectre montrent un très fort couplage.

[Power spectra of two sequences of Barkhausen jumps recorded simultaneously on the two opposite legs of the frame. The comparison of the two spectra shows the existence of a very strong coupling.]

$$
\times
$$

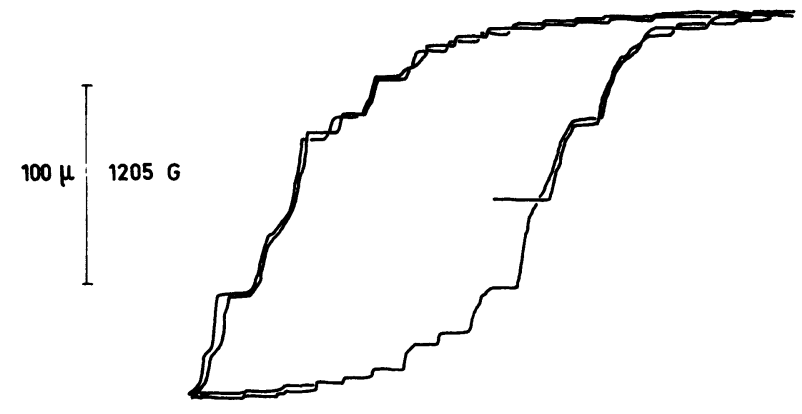

$$
5 m \alpha
$$

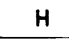

Fig. 13. - Deux enregistrements successifs d'un même cycle d'hystérésis partiel.

[Two successive recordings of a minor hysteresis loop.]

ne faisant intervenir que quelques sauts irréversibles d'aimantation sur chacune des branches ascendante ou descendante (Fig. 14). En choisissant correctement les limites de variation du champ, il est même possible de faire effectuer à la paroi un seul saut irréversible dans chaque sens, mettant en évidence le cycle d'hystérésis reproductible le plus élémentaire que l'on puisse concevoir dans un matériau massif.

Il ressort de ce qui précède que la propriété de conservativité est vérifée pour des déplacements compris entre une et plusieurs épaisseurs de parois $(\sim 1$ à 10). On peut alors parler de fonction potentiel $V(x)$.

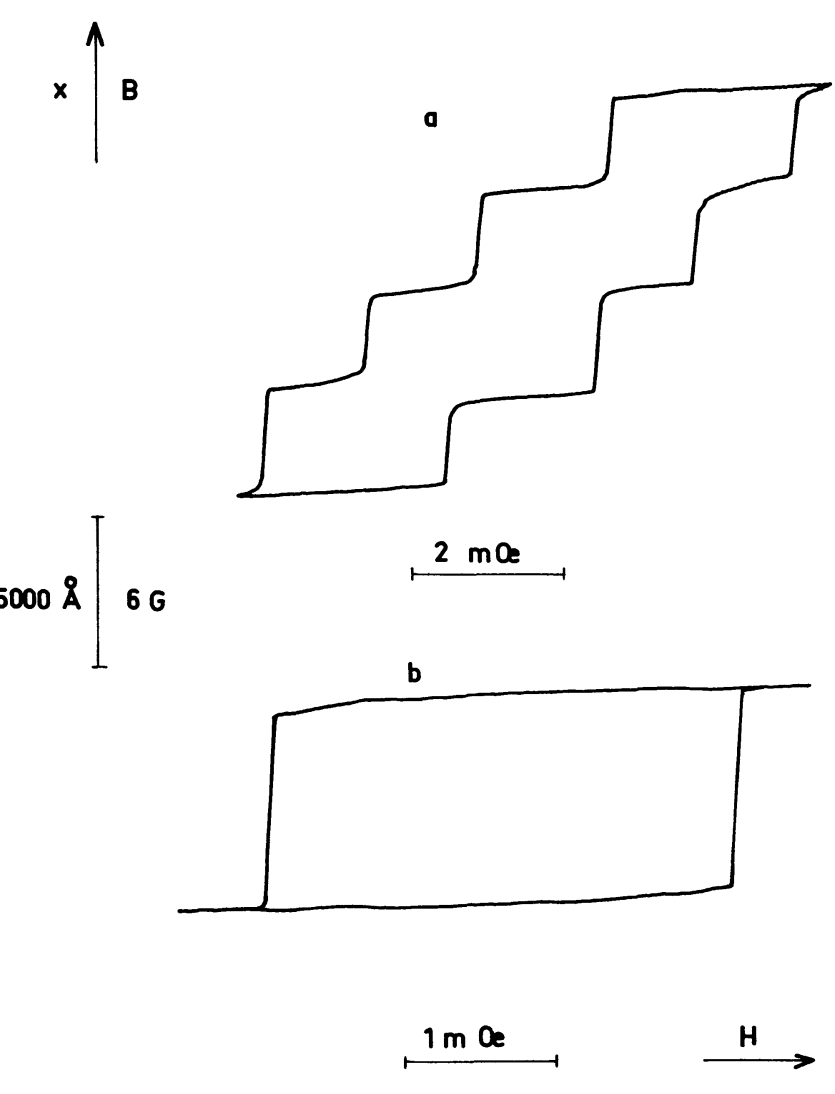

Fig. 14. - a) Cycle d'hystérésis ne faisant intervenir que quelques sauts d'aimantation; $b$ ) Cycle élémentaire décrit entre deux positions d'équilibre stable de la paroi (grain de Preisach).

[a) An hysteresis loop with only a few magnetization jumps; b) An elementary loop between two positions of a stable equilibrium of the wall (Preisach grain).]

Pour voir ce qu'il en était pour des déplacements notables, nous avons fait décrire plusieurs fois le même trajet à la paroi dans les mêmes conditions et nous avons enregistré les séquences de sauts correspondantes. Nous avons ensuite comparé l'autospectre d'une séquence à l'interspectre de deux séquences successives. La figure 15 montre que l'énergie relative

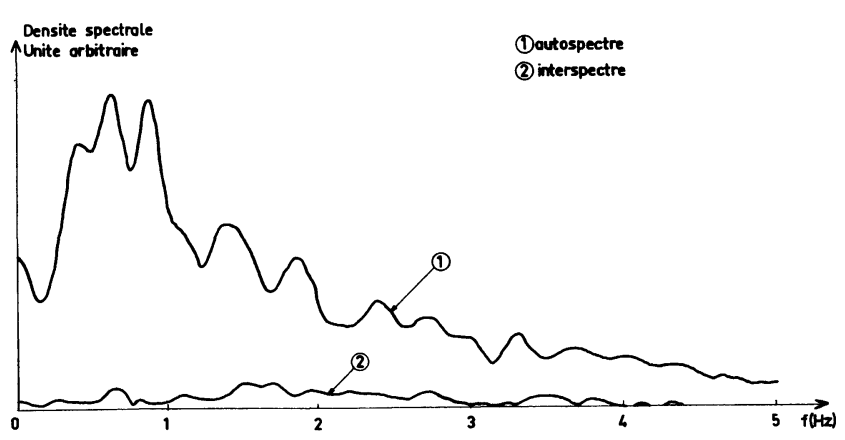

Fig. 15. - Comparaison de l'autospectre d'une séquence de sauts à l'interspectre de deux séquences successives. Ces séquences correspondent à un même déplacement notable de la paroi. L'examen des spectres montre que les séquences sont pratiquement indépendantes.

[Comparison of the autospectrum of a sequence of jumps with the cross-spectrum of two successive sequences, recorded during the same large-amplitude displacement of the wall.] 
à l'interspectre est très faible devant l'énergie mise en jeu dans l'autospectre. Nous en concluons que les séquences de sauts successives correspondant à un même parcours de la paroi sont pratiquement indépendantes. La propriété de conservativité n'est plus vérifiée pour des déplacements notables. Nous avons donné une interprétation de ces faits dans un modèle d'hystérésis développé par J. L. Porteseil $[8,9]$.

3.2.2.3 Reconstitution de la fonction potentiel, ses propriétés statistiques. - Le procédé d'asservissement décrit ci-dessus permet de décomposer le déplacement du point $M$ sur la courbe $V(x)$ en mouvements élémentaires alternativement réversibles et irréversibles. La figure 16 donne un exemple des variations temporelles du courant magnétisant $i$, ou encore du champ $H$


Fig. 16. - Variations temporelles du courant magnétisant qui contrôle les déplacements de la paroi.

[Time dependence of the magnetizing current which controls the motion of the wall.]

qui lui est proportionnel. Les déplacements réversibles correspondent aux arcs de courbe du type $\alpha \beta$ (Fig. 17) : sur ces arcs, le courant $i$ et le champ $H$ croissent régulièrement de manière à déplacer la paroi sur une partie concave vers le haut de la courbe $V(x)$. On a donc accès expérimentalement à la relation qui existe entre la position $x$ de la paroi et le champ $H$ nécessaire pour atteindre cette position. En d'autres termes, on connait sur cet arc de courbe la variation de la fonction énergie potentielle par unité de surface de la paroi :

$$
V(x)=2 J_{\mathrm{s}} \int H(x) \mathrm{d} x .
$$

Par contre, lorsqu'un point d'inflexion est atteint, un saut irréversible se déclenche et l'asservissement impose une diminution brusque du champ magnétisant (segment $\beta \gamma$ ) qui est proportionnelle à la variation de flux enregistrée, et par conséquent à l'amplitude
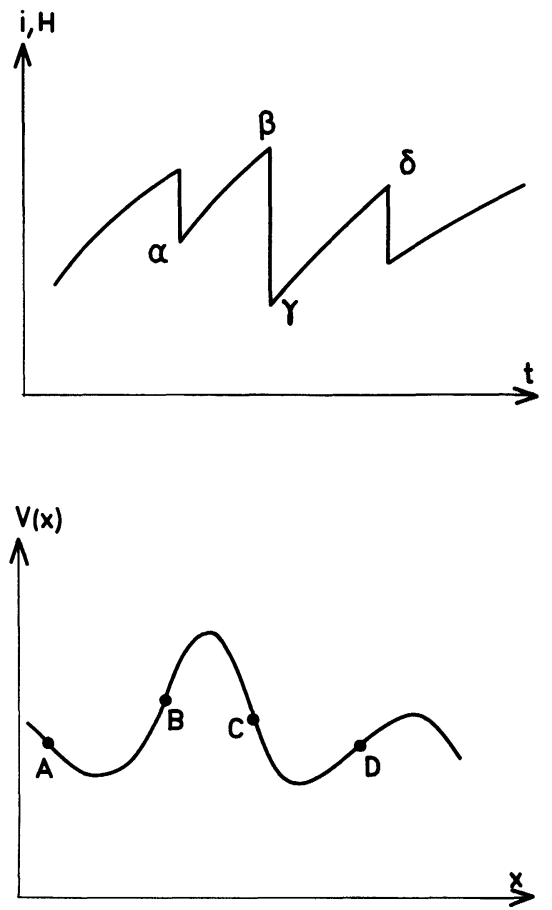

Fig. 17. - Relation entre les fluctuations du courant d'asservissement et les variations de l'énergie potentielle $V(x) ; \alpha \beta$ : déplacements réversibles sur les arcs à concavité positive $(\mathrm{AB}) ; \beta \gamma$ : déplacements irréversibles sur les arcs à concavité négative (BC).

[Relation between the current in the feedback loop and the potential energy $V(x) ; \alpha \beta$ : reversible motion on the regions of positive concavity $(\mathrm{AB}) ; \beta \gamma$ : irreversible motion on the regions of negative concavity $(\mathrm{BC})$.]

du saut. On détermine la constante de proportionnalité grâce à une impulsion de flux calibrée. Le mouvement irréversible fait perdre l'information $H(x)$ et le problème se pose de reconstituer les variations correspondantes de la fonction $V(x)$ en les raccordant avec les parties réversibles adjacentes $\mathrm{AB}$ et $\mathrm{CD}$. Un tel problème a une infinité de solutions si l'on n'impose aucune contrainte particulière à la fonction $V(x)$. On remarque toutefois que sur les arcs réversibles du type $\alpha \beta$, les variations de $H(t)$ sont pratiquement linéaires. Il en est donc de même des variations de $H(x)$ puisque l'asservissement impose alors à la paroi de se déplacer à une vitesse constante. Par conséquent, on peut approcher assez correctement la fonction $V(x)$, proportionnelle à $\int H(x) \mathrm{d} x$, par une succession d'arcs de parabole que l'on raccordera aux points d'inflexion en imposant la continuité de la dérivée $\mathrm{d} V / \mathrm{d} x$. Cette approximation, que suggère ici l'expérience, est postulée dans le modèle de Néel. Dans ces conditions, on peut reconstituer la fonction $V(x)$; la figure 18 donne un exemple des variations de l'énergie potentielle (rapportée à $1 \mathrm{~cm}^{2}$ de paroi) sur une distance de $1,5 \mu$, soit un peu moins de 10 épaisseurs de paroi $(\delta \sim 2000 \AA)$. 


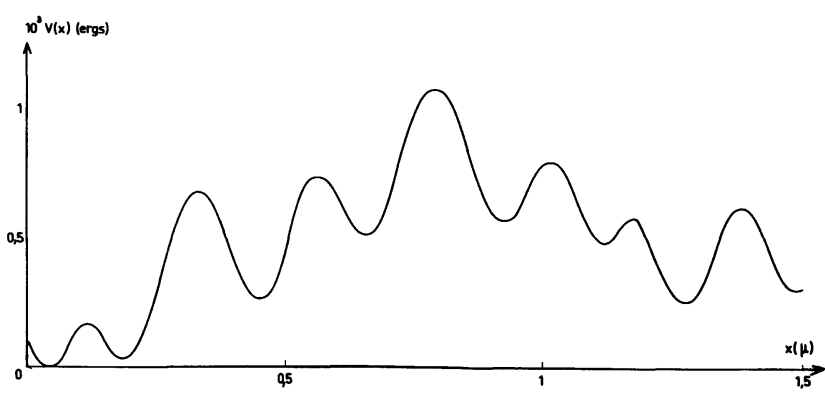

Fig. 18. - Reconstitution de la fonction potentiel $V(x)$ sur une distance de $1,5 \mu$.

[Reconstitution of the potential function $V(x)$ over a distance of $1.5 \mu$.]

On peut, à partir de la fonction potentiel reconstituée, déterminer le champ coercitif

$$
\left(H_{\mathrm{c}}=\frac{1}{2 J_{\mathrm{s}}}\left(\frac{\mathrm{d} V}{\mathrm{~d} x}\right)_{\max }\right)
$$

et la susceptibilité différentielle réversible (terme $a$ de la loi de Rayleigh). Ces deux déterminations sont en excellent accord avec les mesures traditionnelles.

Néel et Bonnet obtenaient une statistique gaussienne pour la densité de probabilité des pentes d'inflexion $p_{i}$ de la courbe $V(x)$. Nous avons trouvé sur un échantillon de 200 pentes, une loi de la forme :

$$
\frac{1}{1+\left(p / p_{0}\right)^{3,5}} \text {. }
$$

Une statistique gaussienne conduirait à des valeurs beaucoup trop faibles du champ coercitif [32].

Le modèle de L. Néel suppose qu'il y a indépendance statistique entre les pentes d'inflexion successives $p_{i}$ alors que Bonnet montre qu'il y a corrélation entre les nombres aléatoires $p_{i}$.

La technique d'asservissement donne accès directement à ces nombres : puisqu'il y a proportionnalité entre les quantités $H$ et $\mathrm{d} V / \mathrm{d} x$, il suffit d'enregistrer la suite des valeurs extrémales du champ magnétique aux points $\beta, \delta$, etc. de la figure 16. On calcule alors sans difficulté le coefficient de corrélation $C_{k}$ des nombres aléatoires $p_{i}$ et $p_{j}$ tels que la différence $j-i$ ait une valeur donnée :

$$
C_{k}=\sum_{i}\left(p_{i}-\bar{p}\right)\left(p_{i+k}-\bar{p}\right) / \sum_{i}\left(p_{i}-\bar{p}\right)^{2} .
$$

La figure 19 donne la variation du coefficient de corrélation déterminé sur un échantillon de 152 valeurs de $p$. On constate que les points expérimentaux s'ajustent de façon très satisfaisante sur la fonction : $C_{k}=\exp (-k / 4,7)$. Ainsi se confirme de façon quantitative ce que laissait prévoir la figure 18 : la fonction aléatoire $V(x)$ possède une mémoire statistique qui s'étend sur une distance de plusieurs épaisseurs de paroi.

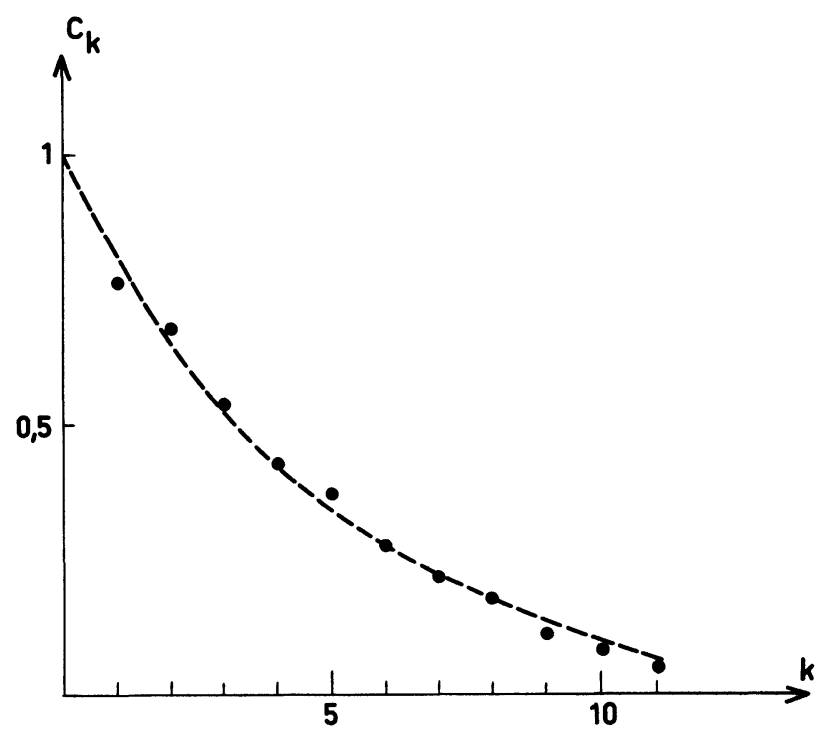

Fig. 19. - Corrélation des pentes d'inflexion successives $p_{i}$ de la fonction $V(x)$. La courbe en pointillé représente la fonction

$$
\exp (-k / 4,7) \text {. }
$$

[Correlation between the inflexion slopes $p_{i}$ of the function $V(x)$. The dotted curve represents the function $\exp (-k / 4.7)$.]

Pour conclure, nous dirons que le modèle de la fonction potentiel interprète bien les faits expérimentaux pour des déplacements qui sont au moins de l'ordre de l'épaisseur de la paroi.

3.2.3 Les faits expérimentaux qui ne sinterprètent pas dans le cadre du modèle de la fonction potentiel. 3.2.3.1 A plus petite échelle, la paroi se comporte comme un objet déformable - En augmentant la sensibilité du fluxmètre, nous avons cherché à préciser la structure fine des cycles élémentaires à un grain décrits plus haut (cf. Fig. 14). Nous avons ainsi obtenu des enregistrements dont la figure $20 a$ donne un exemple. On retrouve sur ce cycle les deux grands sauts ascendant et descendant dont chacun correspond à un déplacement voisin de $6000 \AA$, soit vraisemblablement le franchissement de 3 ou 4 maxima de $V(x)$. Des cycles analogues correspondant au franchissement d'un seul maximum s'obtiennent également sans peine. On observe de plus un très grand nombre d'accidents de bien moindre amplitude. Il est possible de préciser l'allure de ces événements en augmentant encore la sensibilité du fluxmètre (Fig. 20b). Le bruit du fluxmètre est encore négligeable et il s'agit donc bien d'un phénomène magnétique que nous attribuons cette fois à des déformations localisées de la paroi. L'échelle de ces accidents est donnée en déplacements équivalents de la paroi supposée rigide. Alors que les grands sauts sont parfaitement reproductibles d'un cycle à l'autre, les petits ne le sont plus du tout : on ne peut donc plus les décrire à l'aide de la fonction potentiel $V(x)$ qui ne dépend que d'une seule variable d'espace.

A cette échelle d'événements, on trouve une nou- 


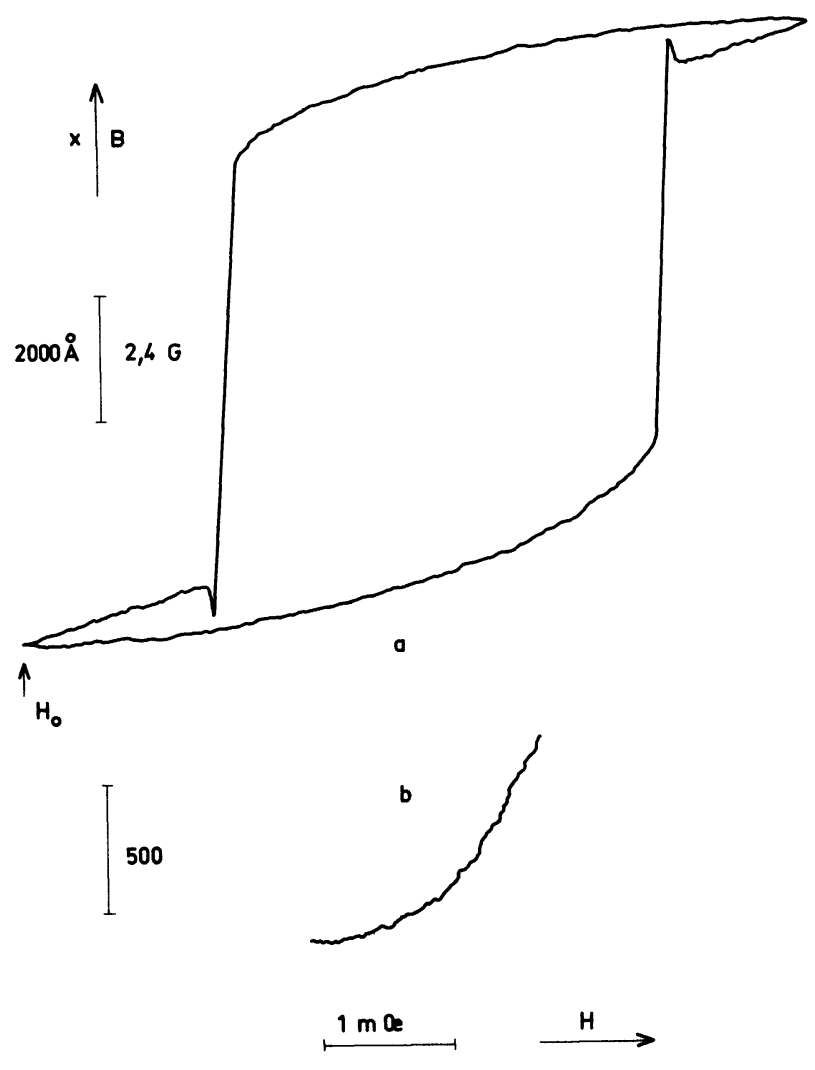

Fig. 20. - a) Enregistrement d'un cycle élémentaire montrant l'existence de petits sauts; b) Enregistrement, à sensibilité plus élevée, des petits sauts sur la branche ascendante du cycle.

[a) Recording of an elementary cycle showing a great number of little magnetization jumps; b) Recording, with a higher sensitivity, of the little jumps on the ascending branch of the loop.]

velle loi de Rayleigh et des cycles d'hystérésis paraboliques (cf. Figs. 20 et 21).

L'étude des petits événements irréversibles exige d'accroître considérablement la sensibilité du flux- mètre et donc d'éliminer les grands sauts qui satureraient la chaîne de mesure. On y parvient grâce à la technique d'asservissement déjà décrite, mais en
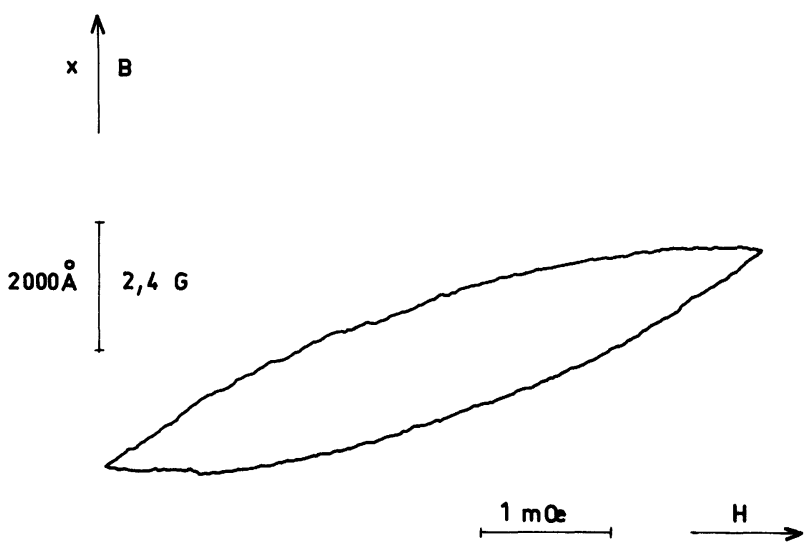

Fig. 21. - Enregistrement d'un cycle de Rayleigh ne faisant intervenir que de petits sauts.

[Recording of a Rayleigh loop with only little jumps.]

appliquant cette fois à la paroi un taux d'asservissement nettement plus élevé. Ce procédé permet alors de contrôler le déplacement de la paroi sur les arcs de la fonction $V(x)$ dont la concavité est tournée vers le bas (arcs instables). Dans ces conditions, on freine les déplacements en bloc de la paroi dès qu'ils se déclenchent et on les rend entièrement réversibles, supprimant ainsi les brusques variations de champ du type $\beta \gamma$ (Fig. 17). Par contre, étant donné que l'asservissement ne réagit que sur un seul paramètre macroscopique qui est le champ magnétisant, on ne peut contrôler les événements irréversibles correspondant aux degrés de liberté internes de la paroi. La figure 22 reproduit un enregistrement ainsi obtenu sur lequel on observe un très grand nombre de petits sauts.
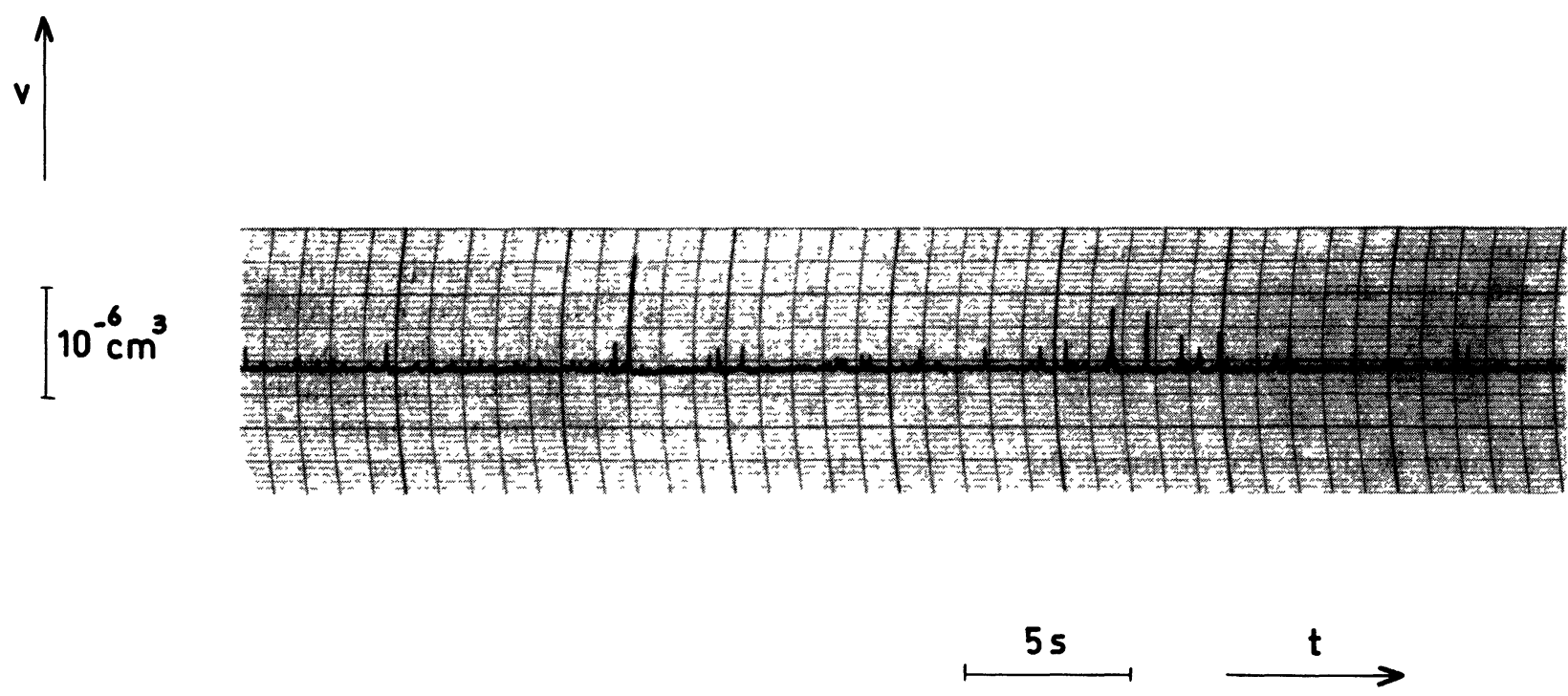

Fig. 22. - Bruit de Barkhausen enregistré en déplaçant la paroi à vitesse constante avec un fort taux de contre-réaction.

[Barkhausen noise recorded by moving the wall at a constant speed with a strong feedback.] 
Nous avons étudié les caractéristiques statistiques du bruit Barkhausen correspondant à ces événements. La probabilité en fonction du volume est de la forme $p(v) \sim \exp \left(-v / v_{0}\right)$ (Fig. 23) où $v_{0}$ est fonction de la température.

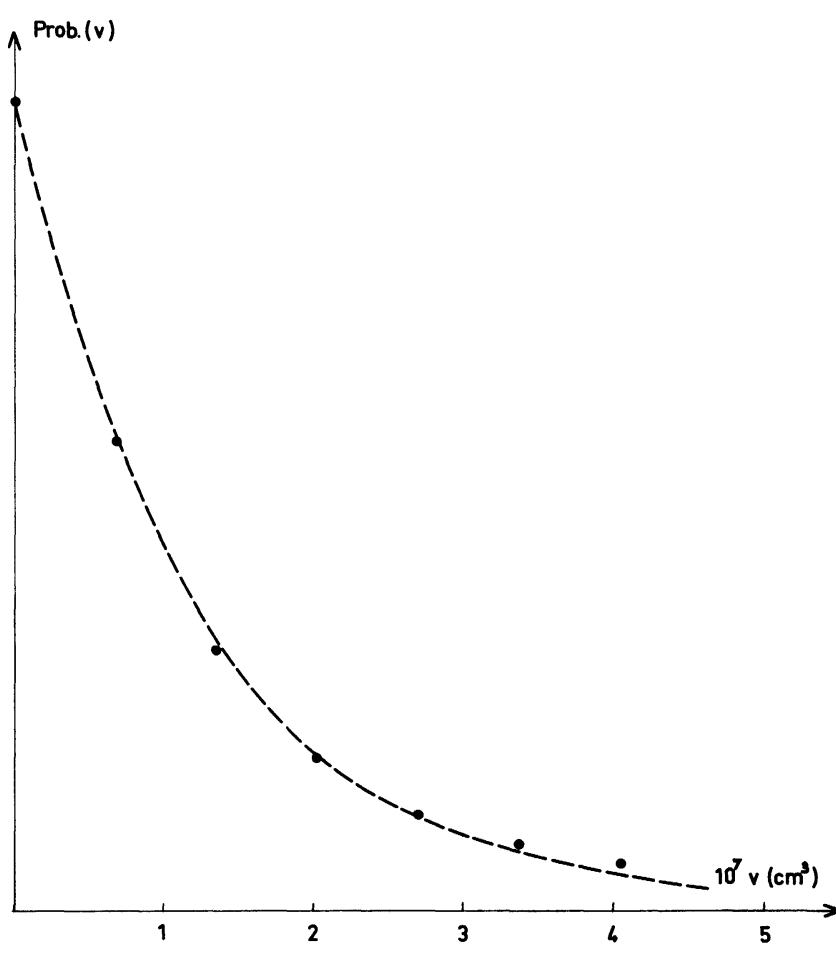

Fig. 23. - Probabilité d'apparition des petits sauts de Barkhausen en fonction de leur volume $v$. La courbe en pointillé représente la fonction $\exp \left(-v / v_{0}\right)\left(v_{0}=1,24 \times 10^{-7} \mathrm{~cm}^{3}\right)$.

[Probability of the little Barkhausen jumps as a function of their volume $v$. The dotted curve represents the function $\exp \left(-v / v_{0}\right)$ $\left.\left(v_{0}=1.24 \times 10^{-7} \mathrm{~cm}^{3}\right).\right]$

L'étude des histogrammes d'intervalles de temps entre les instants d'occurrence des sauts montre l'indépendance statistique de ceux-ci. Ils suivent une - loi de Poisson avec un intervalle de temps moyen entre deux sauts qui vaut, au voisinage de l'ambiante, 266 ms. L'étude de la densité spectrale énergétique du bruit ferromagnétique, effectuée dans les mêmes conditions, recoupe ce résultat. En particulier, la fréquence pour laquelle la puissance de bruit est maximale est très voisine de $1 / \Delta t$. De plus, nous n'avons pas pu mettre en évidence de corrélation entre le volume d'un saut et l'intervalle de temps qui le suit.

3.2.3.2 Transition entre les différents régimes de la paroi : influence de la vitesse et de la température. La figure 24 représente l'évolution, en fonction de la vitesse moyenne $\dot{x}$ de la paroi, d'un cycle d'hystérésis de très faible amplitude. L'excursion de champ et la température $(\sim 300 \mathrm{~K})$ restent constantes pour les cinq enregistrements reproduits. Aux faibles vitesses (24a), seuls interviennent les petits sauts correspondant à des événements localisés sur la paroi. Pour une
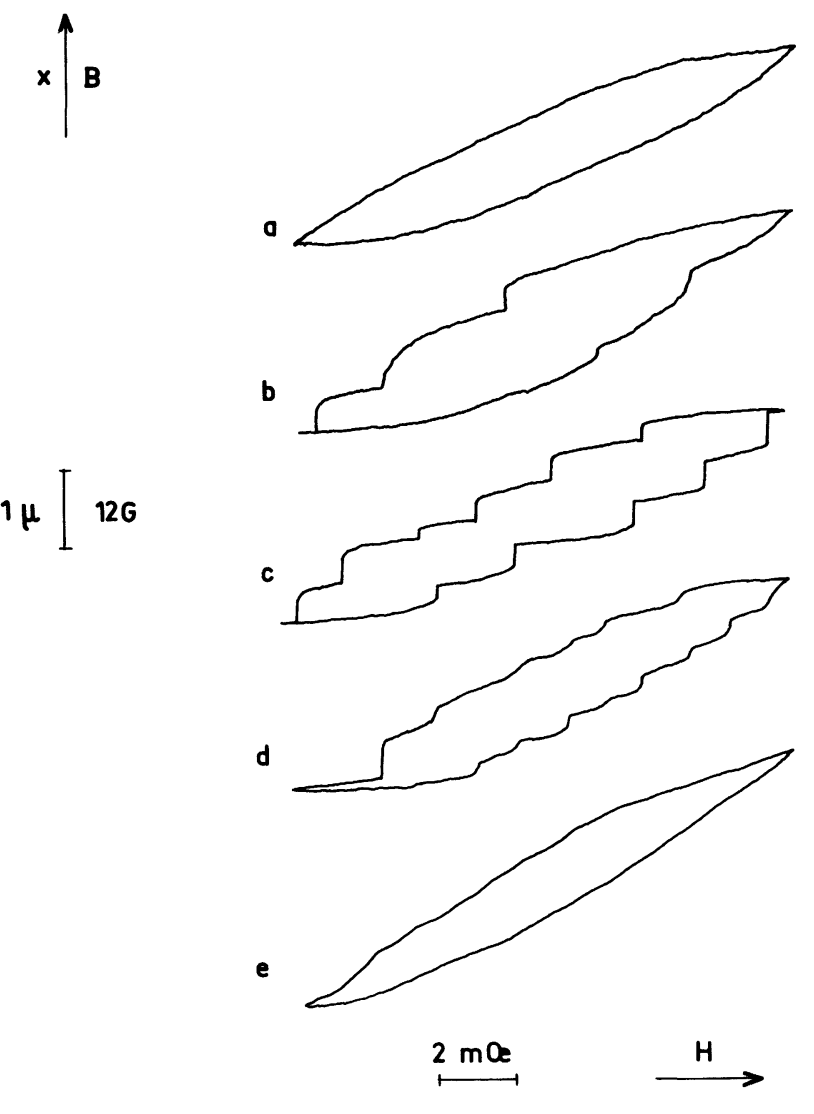

Fig. 24. - a) b) c) d) e) Evolution de la forme des cycles en fonction de la vitesse moyenne de la paroi à la température ambiante.

[a b) c) d) e) Dependence of the shape of the loops on the average velocity of the wall at room temperature.]

vitesse critique $\dot{x}_{1}$, apparaissent quelques sauts de grande amplitude (24b) qui deviennent reproductibles d'un cycle à l'autre dans toute une gamme de vitesses (24c). Il s'agit cette fois de sauts affectant l'ensemble de la paroi que l'on peut alors considérer comme une surface se déplaçant de façon corrélée dans tout l'échantillon. Pour une deuxième vitesse critique $\dot{x}_{2}$, les grands sauts commencent à se subdiviser en petits (24d), ce phénomène s'amplifie lorsqu'on accroît encore la vitesse (24e). Une étude systématique montre que les vitesses critiques $\dot{x}_{1}$ et $\dot{x}_{2}$ dans une région donnée du cristal étudié varient avec la température absolue $T$ comme exp $(-\alpha / T), \alpha$ valant ici $\sim 9280 \mathrm{~K}$. La figure 25 définit, pour une excursion de champ donnée, les domaines du plan $(\dot{x}, T)$ dans lesquels la paroi se déplace suivant les deux régimes décrits plus haut. On observe les mêmes comportements successifs si au lieu d'accroître $\dot{x}$, on diminue $T$. Il est possible de détailler l'apparition des grands sauts au voisinage de la vitesse critique $\dot{x}_{1}$. La figure 26 reproduit trois enregistrements des déplacements de la paroi dans la même région du cristal, pour trois vitesses croissantes de description des cycles à une température constante (on observerait la même succession de phénomènes si on faisait décroître la tempéra- 


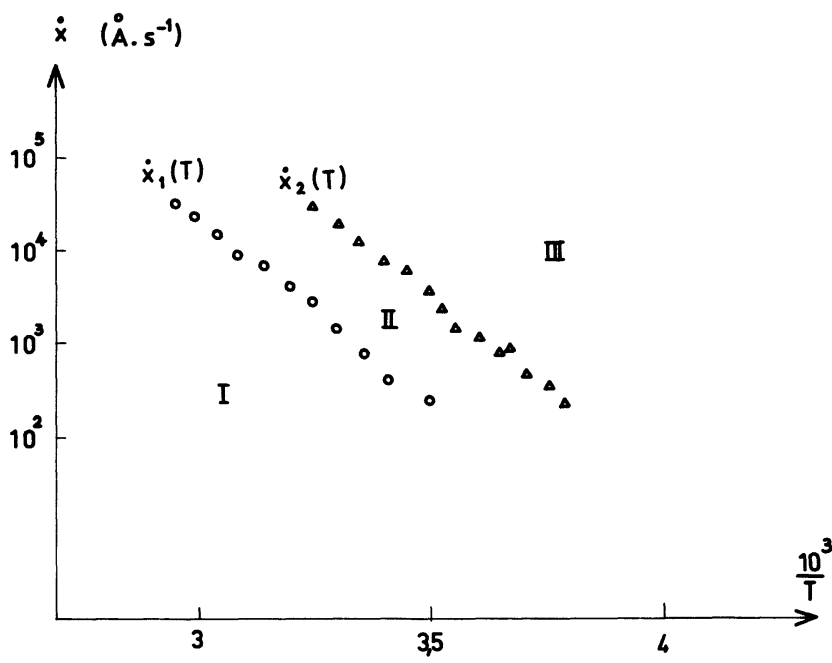

Fig. 25. - Division du plan $(\dot{x}, T)$ en trois régions par les courbes $\dot{x}_{1}(T)$ et $\dot{x}_{2}(T)$. La région II est le domaine d'existence des grands sauts.

[Division of the $(\dot{x}, T)$ plane into three regions by the curve $\dot{x}_{1}(T)$ and $\dot{x}_{2}(T)$. Region II is the domain of existence of the large jumps.]
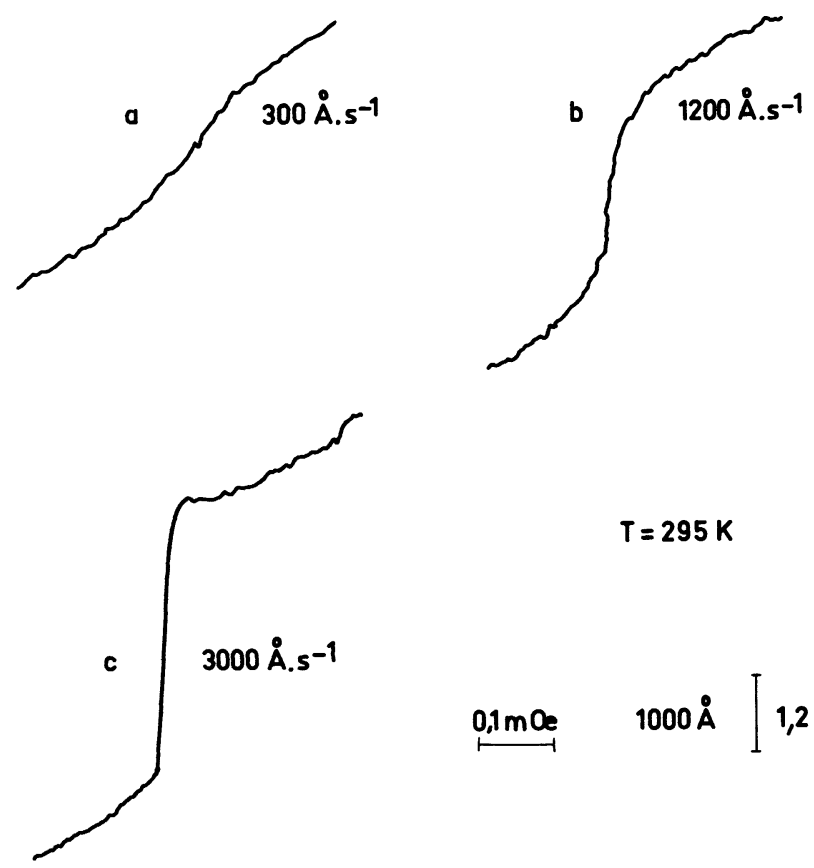

$T=295 K$

Fig. 26. - Apparition des grands sauts en fonction de la vitesse de la paroi.

[Onset of large jumps as a function of wall velocity.]

ture à vitesse constante). La courbe d'aimantation devient de plus en plus abrupte, puis apparaît un grand saut irréversible pour une valeur $H_{0}$ du champ magnétique. Juste avant l'apparition de ce grand saut, la variation du champ $H-H_{0}$ est proportionnelle au cube de la variation d'aimantation $J-J_{0}$ (Fig. 27).

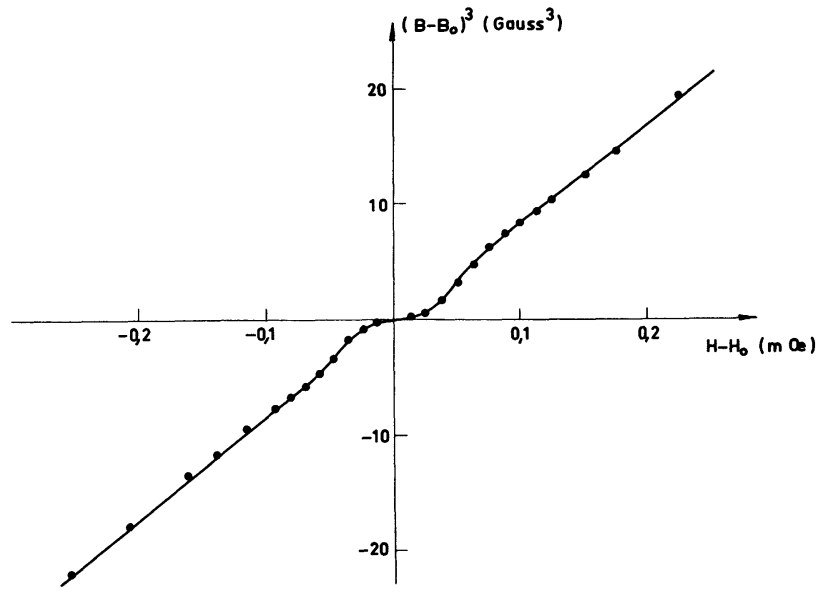

Fig. 27. - Relation entre $H$ et $J$ à la vitesse critique $\dot{x}_{1}(T)$.

[Relationship between $H$ and $J$ at the critical velocity $\dot{x}_{1}(T)$.]

On constate toutefois une déviation de cette loi au voisinage immédiat du point $\left(H_{0}, J_{0}\right)$. On peut également analyser la disparition des grands sauts au voisinage de la vitesse critique $\dot{x}_{2}$. La figure 28 reproduit trois enregistrements du bruit Barkhausen correspondant aux cycles $c, d, e$ de la figure 24. Dans l'enregistrement $28 c$, on observe une succession d'impulsions bien distinctes et reproductibles lorsque la paroi balaie plusieurs fois la même région du cristal. Ces impulsions correspondent aux grands sauts reproductibles de la figure $24 c$. Au voisinage de la vitesse critique $\dot{x}_{2}$ (enregistrement $28 d$ ), la séquence d'impulsions devient beaucoup plus confuse et moins reproductible d'une expérience à l'autre. Pour des vitesses supérieures (enregistrement 28e), le bruit devient tout à fait chaotique. Ces modifications qualitatives sont confirmées par l'évolution du spectre de puissance du bruit Barkhausen (Fig. 29). Le spectre $29 c$ se réduit pratiquement à un pic; des fréquences supplémentaires apparaissent au fur et à mesure que la vitesse croît (spectres $29 d$ et $e$ ).

Nous nous proposons de discuter brièvement ces résultats et de montrer que le déplacement irréversible de la paroi de Bloch présente certains caractères d'une instabilité thermodynamique.

L'irréversibilité des processus d'aimantation est due aux défauts du réseau cristallin qui constituent autant d'obstacles au déplacement de la paroi. L'application d'un champ magnétique permet de franchir les barrières d'énergie correspondantes. D'autre part, l'étude sur le même échantillon du traînage de fluctuations thermiques [10] a montre que ces barrières, valant typiquement $1000 \mathrm{~K}$, peuvent également être franchies par activation thermique. On doit donc envisager un spectre de constantes de temps d'activation $\tau$ qui dépendent bien entendu de la température. Il faut comparer ces constantes de temps à une durée caractéristique qui est le temps nécessaire 



$10 s$

Fig. 28. - Evolution du bruit Barkhausen à des vitesses croissantes.

[Evolution of Barkhausen noise at increasing velocity.]

pour que la paroi, supposée animée d'une vitesse constante, sorte du rayon d'action d'un défaut donné. Nous définirons la durée $\theta$ caractérisant une expé- rience comme le temps que met la paroi à franchir une distance égale à son épaisseur $(2000 \AA \AA$ environ dans ce matériau). 

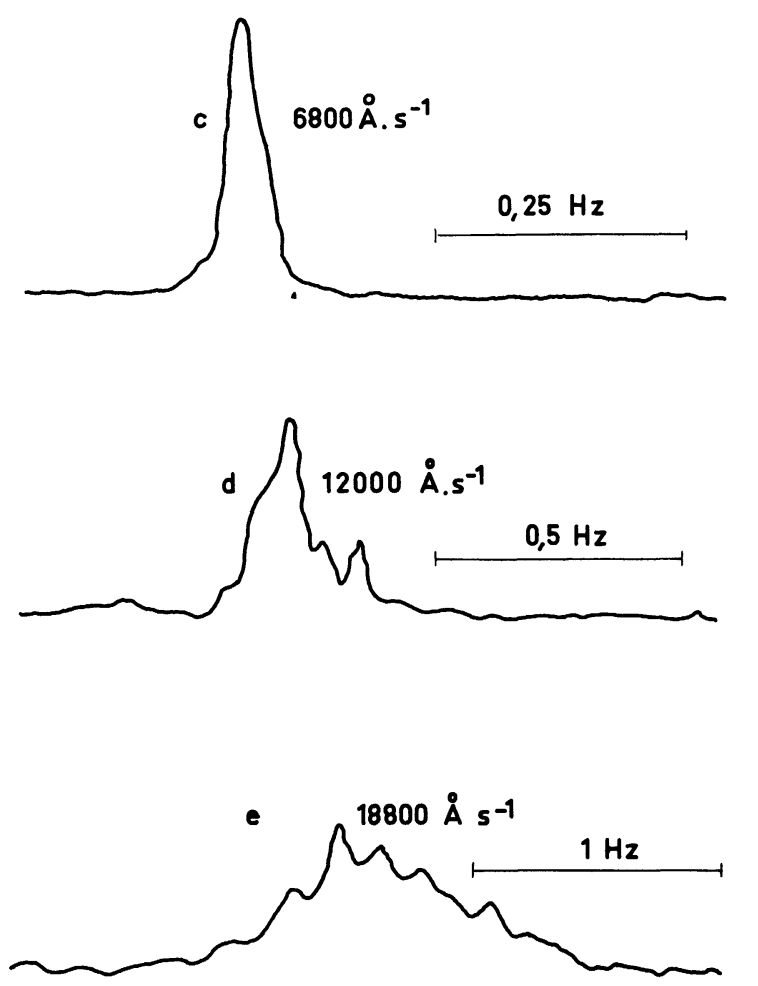

Fig. 29. - Le spectre de puissance du bruit Barkhausen en fonction de la vitesse.

[Power spectrum of the Barkhausen noise as a function of velocity.]

Aux faibles vitesses, $\theta$ est supérieure à toutes les constantes de temps $\tau$. La paroi a le temps de franchir par activation thermique tous les obstacles qu'elle rencontre. L'expérience montre qu'elle progresse alors à petite échelle, par une succession de sauts localisés et aléatoires obéissant à des lois statistiques simples (cf. § 3.2.3.1).

$\mathrm{Au}$ voisinage de la vitesse critique $\dot{x}_{1}$, la plus grande constante de temps $\tau$ devient comparable à $\theta$. Le franchissement de la barrière correspondante par activation thermique devient alors plus difficile : la paroi a tendance à rester accrochée sur cet obstacle, tout en continuant à progresser à petite échelle par ailleurs. Il apparaît vraisemblablement une courbure de la paroi entre les obstacles les plus forts, et par suite des interactions entre ses divers éléments (énergies superficielle et magnétostatique). Lorsque le champ magnétique atteint la valeur qui permet au morceau de paroi mis en jeu de franchir l'obstacle, l'événement a tendance à se propager aux régions voisines de la paroi grâce aux couplages. Pour préciser ce point, nous avons étudié sur les séquences de sauts, la corrélation $C$ entre le volume $v$ d'un saut et l'intervalle de temps $\Delta t$ qui le sépare du saut suivant. Pour les faibles vitesses, on trouve $C \sim 0$ : lorsqu'on augmènte $\dot{x}, C$ devient négative et son module croît jusqu'à 0,4 environ. Les sauts les plus importants favorisent l'apparition des sauts suivants. Ainsi pourrait s'expliquer l'aspect de phénomène critique indiqué par la figure 27. La relation cubique entre l'aiman- tation et le champ pour la vitesse critique suggère de décrire les phénomènes par un formalisme de champ moyen (Landau), sauf au voisinage immédiat du point $\left(H_{0}, J_{0}\right)$.

D'un autre point de vue, on pourrait considérer un grand saut comme une instabilité qui apparaît lorsque les possibilités de dissipation à petite échelle deviennent insuffisantes par rapport à la vitesse imposée à la paroi. On remarque d'ailleurs que le coefficient $b$ de la loi de Rayleigh, qui mesure la dissipation, est environ dix fois plus grand dans le régime des grands sauts que dans celui des petits sauts.

Au-dessus de la vitesse critique $\dot{x}_{1}$, les couplages sont assez forts pour imposer à toute la paroi un comportement corrélé. Un seul paramètre, qui est l'abscisse de son plan moyen, suffit alors à décrire de façon satisfaisante ses déplacements : la gamme des vitesses comprises entre $\dot{x}_{1}$ et $\dot{x}_{2}$ est la région de validité du modèle dit de la fonction potentiel $V(x)$. Cette gamme correspond d'ailleurs à des durées caractéristiques $\theta$ s'échelonnant entre 1 et $0,1 \mathrm{~s}$ environ, alors que les constantes de temps d'activation des grands sauts $\tau$ sont de l'ordre de $1 \mathrm{~s}$ à la température ambiante [10]. Les barrières d'énergie correspondantes valent plusieurs milliers de $\mathrm{K}$.

Aux vitesses plus élevées, des constantes de temps de plus en plus nombreuses deviennent supérieures à $\theta$. Les phénomènes décrits ci-dessus se reproduisent, sur des défauts de plus en plus proches les uns des autres. Les sauts corrélés à grande échelle ont tendance à disparaître, et un régime chaotique apparaît, caractérisé par un spectre de plus en plus complexe. La corrélation entre $v$ et $\Delta t$ décroît jusqu'à $-0,14$ pour la vitesse la plus élevée que nous ayons étudiée. Un tel comportement présente des analogies avec l'écoulement turbulent d'un fluide.

3.2.3.3 Evolution du cycle rectangulaire élémentaire en fonction du nombre de cycles décrits. Quand, après avoir formé un cycle élémentaire stable, on le décrit à vitesse et température constantes, on observe en fonction du nombre de cycles, une fluctuation du champ de déclenchement et une évolution monotone de la valeur moyenne de celui-ci. Les fluctuations du champ de déclenchement sont gaussiennes et sa valeur moyenne croît comme $(\log n)^{1 / 2}, n$ étant le nombre de cycles décrits. Ce phénomène s'interprète simplement en considérant que pour une position donnée du plan moyen de la paroi, il existe un très grand nombre de configurations d'accrochage possibles sur les défauts du réseau. Les énergies de ces configurations sont très voisines et l'agitation thermique permet un choix aléatoire à chaque passage de la paroi. Le champ d'interaction entre les divers éléments de la paroi dépend de la configuration choisie, ce qui entraîne une fluctuation du champ de déclenchement. L'évolution se fait de manière à ce que la paroi soit de mieux en mieux accrochée, ce qui entraîne la croissance des champs de déclenchement. 
Le système s'adapte aux contraintes qui lui sont imposées.

La statistique gaussienne, et la loi en $(\log n)^{1 / 2}$ qui en découle, reflète l'intervention d'un très grand nombre de contributions aléatoires dans les mécanismes d'aimantation des matériaux massifs, même au niveau d'une paroi de Bloch isolée.

Un tel comportement n'est pas nouveau, il est analogue à la reptation des cycles dissymétriques d'un matériau polycristallin $[12,14,8]$. On l'a également observé sur des supraconducteurs; il est lié cette fois à la réorganisation des lignes de flux dans le matériau [15]. Il semble que la reptation soit une caractéristique générale des systèmes doués d'hystérésis soumis à des variations cycliques d'un paramètre macroscopique et dans lesquels des degrés de liberté interne permettent d'effectuer des choix entre de nombreuses configurations.

4. Retour au modèle de la fonction potentiel. Le paragraphe précédent a été consacré au comportement d'une seule paroi de Bloch à $180^{\circ}$ se déplaçant dans un milieu monocristallin aléatoirement perturbé. Nous avons maintenant les éléments nous permettant de faire une analyse critique du modèle de la fonction potentiel. Nous rappellerons d'abord l'essentiel du travail de Bonnet [5] en en dégageant les hypothèses physiques.

Contrairement à Néel [3], Bonnet évite de choisir a priori un modèle pour la fonction potentiel. Il le déduit d'une étude statistique de celle-ci dans le cadre général découlant des phénomènes à l'échelle microscopique qui régissent les fluctuations énergétiques au sein du cristal. On fait les hypothèses suivantes : les déplacements de paroi restent faibles comparés à l'épaisseur de celle-ci, au cours de ceux-ci, la paroi plane en moyenne se comporte comme une membrane fixe en translation et les grandeurs macroscopiques qui la décrivent restent stables. Les perturbations envisagées sont à petite échelle, leur distribution est homogène dans toute la substance et on néglige les effets de bord (joints de grains et limites du cristal). Il en résulte que les fonctions de points qui les représentent sont aléatoires, stationnaires et tridimensionnelles. Sont prises en compte : les contraintes locales (et les déformations), les fluctuations de l'énergie magnétocristalline et de l'énergie d'échange et les inclusions (impuretés, lacunes).

Le calcul de l'énergie superficielle $V(x)$ se fait en plusieurs étapes. On ramène d'abord le problème à une dimension par intégration des perturbations locales $\Theta^{k}(x, y, z)$ dans le plan de la paroi ce qui conduit pour chacune d'elles à une fonction aléatoire monodimensionnelle $\eta^{k}(x)$. On montre ensuite que $V(x)$ se met sous la forme d'un produit de convolution :

$$
V_{x}=\sum_{1}^{k}\left(R_{k} * \eta^{k}\right)(x)=\sum_{1}^{k} V_{k}(x)
$$

D'un point de vue physique, $R_{k}$ représente la modification de la loi angulaire régissant la variation des moments dans la paroi sous l'influence de la perturbation à laquelle correspond $\eta^{k}$. Formellement, ce produit de convolution traduit un filtrage linéaire par effet d'épaisseur. Chaque composante $\eta^{k}$ subit l'action d'un filtre linéaire $\mathcal{F}_{k}$, de réponse impulsionnelle $\mathcal{R}_{k}$, pour fournir la composante $V_{k}$. On somme ensuite tous les $V_{k}$. En d'autres termes, la paroi, du fait de son épaisseur, intègre chacune des perturbations.

La partie fluctuante de $V(x)$ est :

$$
\tilde{V}(x)=V(x)-E\{V(x)\}
$$

$E\{V(x)\}$ est déterminé par un calcul de variations classique et diffère très peu de ce que serait l'énergie superficielle dans le monocristal parfait. Cela tient au fait physique suivant : l'énergie superficielle est essentiellement déterminée par l'énergie magnétocristalline et l'énergie d'échange qui sont toujours grandes devant les énergies dues aux perturbations.

On définit ensuite les fonctions de corrélation à partir du tenseur de covariance $\Lambda^{k l}\left(\mathbf{M}, \mathbf{M}^{\prime}\right)$ des composantes centrées $\tilde{\Theta}^{k}=\Theta^{k}-E\left\{\Theta^{k}\right\}$. On a $\Lambda^{k l}=E\left\{\tilde{\Theta}^{k}(\mathbf{M}) \tilde{\Theta}^{l}\left(\mathbf{M}^{\prime}\right)\right\}$. Compte tenu de l'hypothèse de stationarité, $\Lambda^{k l}$ ne dépend que de la différence $\mathbf{M}-\mathbf{M}^{\prime}$. De là, on déduit l'expression de la matrice de corrélation $C^{k l}(\tau)$ des $\tilde{\eta}^{k}$ :

$$
C^{k l}(\tau)=E\left\{\tilde{\eta}^{k}(x) \tilde{\eta}^{l}(x-\tau)\right\}
$$

où

$$
\tilde{\eta}^{k}=\eta^{k}-E\left\{\eta^{k}\right\}
$$

et par sommation, la fonction de corrélation $\Gamma(\tau)$ de $V(x)$.

On a :

$$
\Gamma(\tau)=E\{\tilde{V}(x) \tilde{V}(x-\tau)\}
$$

qui s'écrit, en tenant compte de l'expression de $V(x)$ :

$$
\sum_{k, l} \int_{-x}^{+x} \int_{-x}^{+x} C^{k l}(\lambda) R_{k}(\mu) R_{l}(\lambda+\mu-\tau) \mathrm{d} \lambda \mathrm{d} \mu .
$$

La fonction de corrélation de l'opposition $\Omega(x)$, c'est-à-dire de $\mathrm{d} V / \mathrm{d} x$ qui est la force qui s'oppose au déplacement de la paroi, s'en déduit immédiatement. On a :

$$
\Psi(\tau)=E\{\Omega(x)-\Omega(x-\tau)\}=-\frac{\mathrm{d}^{2}}{\mathrm{~d} \tau^{2}} \Gamma(\tau) .
$$

Introduisons dès à présent une représentation en en fréquences spatiales à l'aide des transformées de Fourier ci-dessous :

$-r_{k}(v) \stackrel{\text { T.F. }}{\rightleftarrows} R_{k}(x)$, le gain complexe du filtre $\mathcal{F}_{k}$; 
$-g_{y}(v) \stackrel{\text { T.F. }}{\rightleftarrows} \Gamma(\tau)$, densité spectrale de l'énergie superficielle $V(x)$;

$$
-g(v) \stackrel{\text { T.F. }}{\rightleftarrows} \Psi(\tau) \text { avec } g(v)=4 \pi^{2} v^{2} g_{i}(v) \text {. }
$$

Pour aller plus loin, il faut faire des hypothèses complémentaires relatives à l'étendue de la mémoire statistique des perturbations. Envisageons d'abord le cas des perturbations à l'échelle microscopique. Ce cas correspond à une mémoire statistique des perturbations faible devant l'épaisseur de paroi, ce qui a pour effet que l'épanouissement des $C^{k l}(\tau)$ est faible devant l'épaisseur de paroi ou encore que les $C^{k l}$ sont très concentrés à l'échelle des $R_{k}$. On a alors :

$$
\Gamma(\tau) \# \sum_{k, l} a^{k l} \Gamma_{k l}(\tau)
$$

avec

$$
\Gamma_{k l}(\tau)=\int_{-x}^{+x} R_{k}(\mu) R_{l}(\mu-\tau) \mathrm{d} \mu
$$

et

$$
a^{k l}=\int_{-x}^{+x} C^{k l}(\hat{\lambda}) \mathrm{d} \hat{\lambda}
$$

Il en résulte que ce sont les seuls paramètres physiques de la paroi qui imposent l'allure de la fonction de corrélation $\Gamma(\tau)$, le rôle des perturbations n'étant que d'en pondérer l'amplitude par l'intermédiaire $\operatorname{des} a^{k l}$.

De cet effet de filtrage, on peut déduire la forme de la fonction d'opposition. Chacun des filtres $\mathcal{F}_{k}$ a une fréquence de coupure $v_{0}^{k}$, ce qui entraîne la possibilité, sans introduire d'erreur sensible, d'attribuer au gain complexe un support borné

$$
\left[-v_{0}^{k}+v_{0}^{k}\right] \text {. }
$$

Si $v_{0}$ est la plus grande des fréquences de coupure de l'ensemble des filtres $\mathcal{F}_{k}$, on peut considérer que $g_{i}(v)=\sum_{k, l} a^{k l} r_{k}(v) r_{l}^{*}(v)$ et par extension $g_{\Omega_{2}}(v)$ ont un support borné $\left[-v_{0}+v_{0}\right]$. Ce qui nous autorise à utiliser le théorème d'interpolation pour construire $\Omega(x)$ à partir d'échantillons de cette fonction pris sur l'axe des $x$ à des intervalles constants égaux à $1 / 2 v_{0}$; chaque échantillon étant pondéré par la fonction d'interpolation :

$$
\frac{\sin 2 \pi v_{0}\left(x-x_{i}\right)}{2 \pi v_{0}\left(x-x_{i}\right)}
$$

Une approximation raisonnable consiste à remplacer les fonctions d'interpolation par des rectangles de même aire. $\Omega(x)$ prend alors l'allure d'une fonction en escalier, dont la primitive $V(x)$ est une fonction en dent de scie. On retrouve le modèle polygonal de Néel.

Envisageons maintenant le cas opposé de perturbations à évolution lente qui interviennent dans les grands déplacements de parois. Ces perturbations possèdent une mémoire statistique très importante comparée à l'épaisseur de paroi. Dans l'expression de $\Gamma(\tau)$, il convient alors de tenir compte d'un ensemble de valeurs $\varepsilon$ de $\lambda$, telles que $C^{k l}(\lambda+\varepsilon)$ soit sensiblement égal à $C^{k l}(\lambda)$ alors que $R_{l}(\lambda+\varepsilon+\mu-\tau)$ est pratiquement nul. On a alors :

$$
\Gamma(\tau) \# \sum_{k, l} C^{k l}(\tau) \int_{-\infty}^{+\infty} R_{k}(u) \mathrm{d} u \int_{-\infty}^{+\infty} R_{l}(v) \mathrm{d} v
$$

$\Gamma(\tau)$ se réduit à une forme linéaire des $C^{k l}(\tau)$, il n'y a plus d'effet de filtrage et plus de fréquence de coupure systématique à l'échelle des déplacements envisagés. Le modèle du contour polygonal pour $V(x)$ n'est plus valable.

Dans le cas des perturbations à échelle microscopique, le modèle permet de préciser les paramètres introduits par Néel. On obtient : $2 l=1 / 2 v_{0}$. La variance de la pente $1 / 2 P_{0}^{2}$ est reliée à la fonction de corrélation de l'opposition :

$$
\frac{1}{2} P_{0}^{2}=\left\{-\frac{\mathrm{d}}{\mathrm{d} \tau^{2}} \Gamma(\tau)\right\}_{\tau=0} .
$$

Cette quantité dépend du type de paroi et de la nature des perturbations. Il justifie également la validité de la statistique gaussienne et introduit contrairement à Néel des corrélations entre pentes successives du modèle polygonal en déterminant $\Gamma(\tau)$ et $\Psi(\tau)$.

L'aspect nouveau de la théorie de Bonnet par rapport au modèle de Néel réside dans le fait de considérer la paroi comme un filtre linéaire pour chaque perturbation. L'effet de filtrage semble toujours exister : il est lié à la structure même de la paroi; par contre, la fréquence de coupure de chaque $\mathcal{F}_{k}$ dépend de l'étendue de la mémoire statistique de chaque perturbation, elle est liée au type de défaut qui en est la cause. Aucun des deux modèles ne tient compte de l'action de l'agitation thermique, par contre, ils supposent tous les deux que la paroi se déplace comme une membrane rigide. L'étude faite au paragraphe précédent nous montre dans quelles conditions de vitesses $(\dot{x})$ et de température, il faut nous placer pour que cette hypothèse soit raisonnable.

Voyons d'abord en quoi divergent le modèle et les résultats expérimentaux. Nous observons : que les déplacements de la paroi se font à une échelle égale ou supérieure à son épaisseur; que la statistique des points d'inflexion de $V(x)$ n'est pas gaussienne; qu'il existe des corrélations entre ces pentes sur quelques épaisseurs de paroi ( $\sim 5$ à 10$)$ et enfin que la propriété de conservativité n'est vérifiée que sur quelques épaisseurs de paroi $(\sim 10)$. L'expérience montre que la fonction opposition est un contour polygonal; ce qui entraîne pour $V(x)$ une succession d'arcs de parabole. Le modèle prévoyait respectivement une fonction en escalier et une fonction en dent de scie. Une statistique gaussienne des pentes d'inflexion 
conduirait à des valeurs trop faibles du champ coercitif. Néel avait d'ailleurs remarqué [3] que l'accord avec l'expérience était bien meilleur avec une statistique analogue à celle que nous trouvons expérimentalement [32]. L'argument de Bonnet pour justifier la statistique gaussienne est dans le cas des défauts à faible mémoire statistique, l'application du théorème central limite. Aux termes de celui-ci, la loi de la somme d'un très grand nombre de variables aléatoires indépendantes converge vers une loi gaussienne. Les divergences sur les autres points sont vraisemblablement dues au fait que la mémoire statistique des perturbations a une étendue qui n'est plus faible devant l'épaisseur de paroi $\delta$, mais de l'ordre de $\delta$ ou de quelques $\delta$.

Dans le monocristal qui a servi à cette étude, nous avons essentiellement trois types de défauts : des inclusions à petite échelle, des dislocations aléatoirement réparties dont l'échelle moyenne est de l'ordre de $50 \mu$ et enfin des sous-joints de grains à l'échelle du millimètre. La désorientation de ces derniers est très faible, elle reste toujours inférieure à la minute. Les inclusions constituent bien des perturbations à échelle microscopique au sens du modèle. Par contre, il ne nous semble pas qu'il en soit de même pour les dislocations isolées. La mémoire statistique de tels défauts est certainement supérieure à l'épaisseur de paroi. Pour s'en convaincre, il suffit de se souvenir que les champs de contrainte qui leur sont associés décroissent (en dehors du cour de la dislocation) en $(r)^{-1 / 2}$. Quant aux sous-joints de grains, ce sont des perturbations à évolution très lente à l'échelle de quelques épaisseurs de parois. Il n'y a pratiquement plus de filtrage linéaire, la fréquence de coupure du filtre linéaire correspondant ayant pour ordre de grandeur $1 / L_{1} ; L_{1}$ étant l'échelle de ces défauts.

Voyons maintenant de façon plus précise ce que peut apporter l'expérience. Il est possible de mesurer $\mathrm{d} V / \mathrm{d} x$ et de reconstituer $V(x)$ au moins sur quelques millimètres, sinon sur toute l'étendue du déplacement de la paroi dans le cristal. On a donc accès à $\Gamma(\tau)$ $\Psi(\tau)$ et les densités spectrales correspondantes dans l'intervalle de fréquences spatiales $1 / L$ à $1 / \delta$ ou à $1 / 2 \delta$, $L$ étant la largeur de l'échantillon. On a également accès à une statistique affinée des pentes d'inflexion et aux corrélations de celle-ci.

5. Autre aspect des phénomènes : étude de la force exercée par les défauts du réseau sur la paroi. Notion d'homothétie interne dans les déplacements d'une paroi de Bloch. - Dans le cas d'un déplacement asservi de la paroi, le champ magnétique fluctuant $h(x)$ qui lui est appliqué pour contrôler à tout instant son déplacement est proportionnel à la force exercée par les défauts du réseau sur la paroi. Dans un modèle de fonction potentiel $h(x)$ est proportionnel à la fonction opposition $\mathrm{d} V / \mathrm{d} x$.

La figure 30 reproduit les valeurs de $h(x)$ relevées par intervalles sur des enregistrements graphiques.

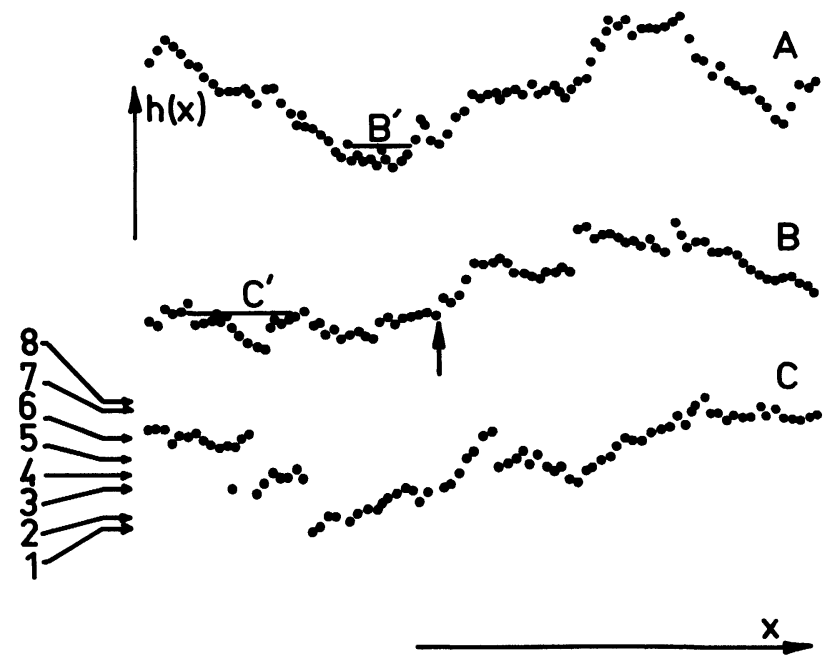

Fig. 30. - Trois enregistrements de $h(x)$ à des échelles différentes. Les longueurs des axes $x$ et $h$ représentent respectivement : $5 \times 10^{-2} \mathrm{~cm}$ et $40 \mathrm{mOe}(\mathrm{A}) ; \quad 5 \times 10^{-3} \mathrm{~cm}$ et $20 \mathrm{mOe}(\mathrm{B})$; $10^{-3} \mathrm{~cm}$ et $10 \mathrm{mOe}(\mathrm{C})$.

[Three recordings of $h(x)$ at different scales. The lengths of the $x$ and $h$ axes are respectively : $5 \times 10^{-2} \mathrm{~cm}$ and $40 \mathrm{mOe}(\mathrm{A})$; $5 \times 10^{-3} \mathrm{~cm}$ and $20 \mathrm{mOe}(\mathrm{B}) ; 10^{-3} \mathrm{~cm}$ and $10 \mathrm{mOe}(\mathrm{C})$.]

Les fragments $\mathrm{A}, \mathrm{B}$ et $\mathrm{C}$ correspondent respectivement à des déplacements de la paroi égaux à $8,5 \times 10^{-2}, 8,5 \times 10^{-3}, 1,7 \times 10^{-3} \mathrm{~cm}$. On remarque la similitude frappante de ces enregistrements les uns avec les autres, tandis que l'échelle à laquelle on observe la fonction $h(x)$ varie d'un facteur 50 entre A et C. En d'autres termes, une partie donnée de cet objet aléatoire reste statistiquement semblable à l'objet tout entier lorsqu'on la dilate convenablement. Cette propriété d'homothétie interne est bien entendu limitée par deux échelles physiques : l'épaisseur de la paroi (environ $2 \times 10^{-5} \mathrm{~cm}$ ) d'une part, son libre parcours dans le monocristal (de l'ordre de $0,1 \mathrm{~cm}$ ) d'autre part.

Un calcul numérique donne accès à la transformée de Fourier $J \in(k)$ de $h(x)$. La figure 31 montre les variations du spectre de puissance $|\cdot \mathscr{H}(k)|^{2}$ en coordonnées logarithmiques. Il est proportionnel à $k^{-2}$, sauf pour les nombres d'onde correspondant à l'épaisseur de paroi où apparait un pic assez net. L'homothétie interne de $h(x)$ se manifeste par un spectre invariant à un facteur près dans un changement d'échelle. Remarquons que la loi de décroissance en $k^{-2}$ est aussi celle d'une marche aléatoire.

Il faut souligner que le spectre de $h$, considéré comme une fonction du temps, a déjà été étudié [16]. Il décroît comme $f^{-2}$ sur une gamme de fréquences de trois décades au moins. Ce résultat est plus général que celui présenté ici, car il reste valable même lorsqu'un modèle d'énergie potentielle ne l'est plus. Il est clair néanmoins que la loi en $k^{-2}$ équivaut à une loi en $f^{-2}$ puisque l'asservissement impose une relation linéaire entre l'abscisse de la paroi et le temps. 


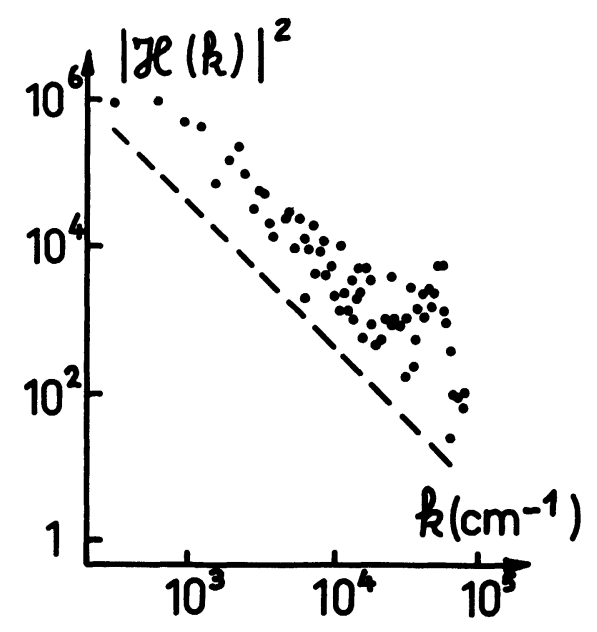

Fig. 31. - Spectre de puissance de $h(x)$. La ligne en pointillés indıque la pente - 2: les ordonnées sont en unités arbitrares.

[Power spectrum of $h(x)$. The dotted lines indicates the slope -2 ; the ordinates are in arbitrary units.]

Les concepts permettant de décrire des objets tels que la courbe $h(x)$ sont bien connus des mathématiciens. Cherchons par exemple les points d'équilibre de la paroi en champ nul. Il s'agit d'étudier les intersections de la courbe $h(x)$ avec l'axe des $x$. Seules les intersections ayant lieu par valeurs croissantes sont des positions d'équilibre stable. Du fait de l'homothétie interne de $h(x)$ ses passages à zéro forment un ensemble de points à structure hiérarchique, chaque intersection se révélant être en fait une séquence d'intersections à plus petite échelle au fur et à mesure que la résolution croît. Si l'on assimile $h(x)$ à une marche aléatoire, comme le suggère l'expérience, on conclut que cet ensemble de points se caractérise par une dimension fractale égale à 0,5 [17]. Le nombre moyen d'intersections compris dans un fragment de longueur $l$ est alors proportionnel à $\sqrt{l}$. On est d'ailleurs conduit à considérer les processus d'aimantation comme des phénomènes aléatoires non stationnaires en général, puisque les propriétés moyennes dépendent explicitement de la longueur des échantillons analysés.

Le problème des déplacements de la paroi de Bloch se traite en remarquant que la paroi soumise à un champ $H$, supposé par exemple croissant, surmonte tous les défauts tels que $h(x)<H$. La figure 32 indique comment la croissance de $H$ fait se modifier les régions de l'enregistrement $\mathrm{C}$ où cette inégalité est vérifiée (régions indiquées en trait fort). De nouveaux segments viennent se connecter au segment initial jusqu'à occuper tout le fragment $\mathrm{C}$. Toute cette évolution n'est en fait qu'un détail des phénomènes analogues qui se reproduiraient à des échelles supérieures sur les enregistrements $B$, puis $A$ (les segments $C^{\prime}$ et $B^{\prime}$ indiqués sur $B$ et $A$ ont respectivement les mêmes longueurs réelles que $C$ et $B$ ). Une telle évolution semble liée aux étapes successives de la construc-

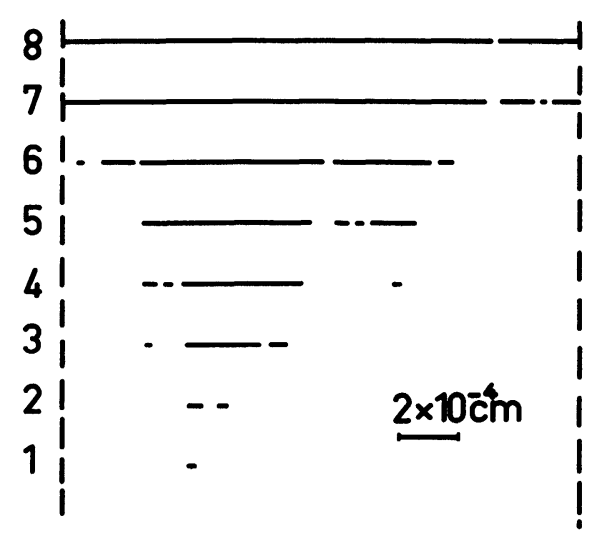

Fig. 32. - Evolution des régions du cristal pouvant contenir la paroi en fonction du champ appliqué $H$. Huit valeurs croissantes de $H$ sont repérées par des numéros de 1 à 8 qui correspondent aux ordonnées indiquées sur la figure 30 . Les longueurs des axes $x$ et $H$ représentent respectivement $: 10^{-3} \mathrm{~cm}$ et $5 \mathrm{mOe}$.

[Evolution of the regions of the crystal which can contain the wall as a function of the applied field $H$. Eight increasing values of $H$ are labelled by the nombers from 1 to 8 , which correspond to the ordinates indicated on figure 30. The lengths of the $x$ and $H$ axes are respectively $10^{-3} \mathrm{~cm}$ and $5 \mathrm{mOe}$.]

tion d'un ensemble aléatoire de type Cantor [17]. Lorsque $H$ croît, la position de la paroi est à chaque instant l'extrémité droite du segment en trait fort qui la contient. Chaque fois qu'un nouveau segment vient se connecter au précédent, la paroi saute brusquement jusqu'à la nouvelle extrémité qu'elle peut atteindre. On reconstitue ainsi facilement la loi de déplacement $x(H)$, composée de sauts irréversibles séparés par des trajets réversibles. La figure 33 donne un exemple de cette loi déterminée sur le fragment B (on n'a tenu compte que des déplacements irréversibles). Ce résultat est à comparer au résultat expérimental de la figure 9. Etant donnée la structure hiérarchique de $h(x)$, on obtiendrait des diagrammes statistiquement semblables à des échelles inférieures ou supérieures.

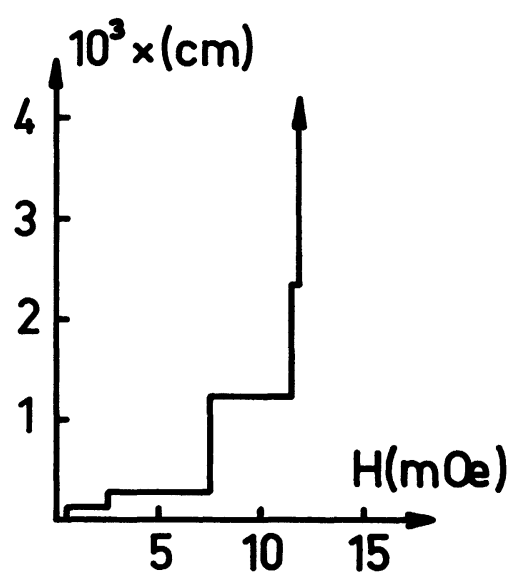

Fig. 33. - Un exemple de loi de déplacement de la paroi soumise à un champ croissant. La flèche sur l'enregistrement B (Fig. 30) indique la position initiale choisie arbitrairement.

[An example of the law of motion of the wall subjected to an increasing field. The arrow on recording B (Fig. 30) indicates the (arbitrary) initial position.] 
Le graphe de $x(H)$ peut être considéré comme une version aléatoire de l'escalier du diable [17].

Remarquons également que ces résultats suggèrent une interprétation des lois de Rayleigh. Admettons en effet que $h$ soit assimilable à la marche aléatoire d'une particule libre, du moins dans une certaine gamme de champs autour de $h=0$. On prévoit que les valeurs de $h(x)$ mesurées sur un échantillon de longueur $l$ obéiront à une loi de probabilité telle que $\left\langle h^{2}\right\rangle \sim l$. Le problème statistique des déplacements de la paroi dans les champs faibles reste semblable à lui-même si l'on applique des facteurs d'échelle $\lambda$, sur l'axe $h$, et $\lambda^{2}$ sur l'axe $x$. On s'attend donc à une variation quadratique de l'espérance mathématique du déplacement de la paroi en fonction du champ appliqué.

6. L'activation thermique et le déplacement de la paroi de Bloch à $\mathbf{1 8 0}^{\circ}$. - Nous nous bornerons ici à donner quelques aspects de cette étude; le lecteur plus particulièrement intéressé pourra se reporter à la référence [19].

Nous avons insisté tout au cours de cet exposé sur l'importance des fluctuations thermiques. Nous nous proposons d'étudier d'une façon plus systématique leur influence sur le déplacement de la paroi de Bloch en nous plaçant dans le cas particulier où celle-ci est soumise à une force presque suffisante pour qu'elle se décroche de tous les obstacles. Pour cela, on lui applique un champ voisin du champ coercitif.

Deux types d'expériences ont été réalisés. L'un a trait à l'étude du traînage de la paroi en fonction du temps et de la température; l'autre à l'évolution du volume moyen des petits sauts de Barkhausen en fonction de la température, quand on impose à la paroi de se déplacer à vitesse constante.

Pour réaliser les expériences de traînage on soumet la paroi à un champ magnétique voisin du champ coercitif $\left(H_{\mathrm{c}}=28 \mathrm{mOe}\right)$ pendant un temps suffisant pour qu'on n'observe plus de déplacement appréciable de celle-ci. On applique alors un petit échelon de champ supplémentaire d'amplitude telle que le déplacement instantané soit de l'ordre de $3 \mu$, soit 15 à 20 fois l'épaisseur de la paroi. Ce premier déplacement très rapide est suivi de mouvements lents dus aux fluctuations thermiques. On mesure la variation d'aimantation entre deux temps $t_{1}$ et $t_{2}\left(t_{2}>t_{1}\right)$ en fonction de $T$ ( $t$ varie de 1 à $25 \mathrm{~s}$ et $T$ de 7 à $320 \mathrm{~K}$ ).

En ce qui concerne le second type d'expériences on utilise la technique décrite au paragraphe 3.2.3.1. Lors du déplacement de la paroi l'asservissement applique un champ magnétique fluctuant au cours du temps suivant les obstacles que rencontre la paroi ; mais toujours voisin du champ coercitif. Le bruit de Barkhausen observé présente les deux caractéristiques suivantes : les instants d'apparition des sauts sont statistiquement indépendants; la probabilité d'observer un saut de volume $v$ obéit à une loi exponentielle : $p(v) \sim \exp \left(-v / v_{0}\right)$. Dans la gamme où nous avons pu mener à bien cette étude, c'est-à-dire entre 253 et $323 \mathrm{~K}$, la loi de variation du volume moyen $v_{0}$ est :

$$
v_{0}=1,7 \times 10^{-11} T^{4,4} \mathrm{~cm}^{3} .
$$

La vitesse de déplacement de la paroi était de $4000 \AA . \mathrm{s}^{-1}$ (soit d'environ deux fois l'épaisseur de paroi par seconde). Cette valeur réalise un compromis intéressant entre deux nécessités contradictoires : d'une part obtenir un nombre important de sauts pendant un temps raisonnable; d'autre part éviter des empiètements trop nombreux entre sauts. On peut développer un certain nombre d'arguments montrant que ces deux types d'expériences ne sont en fait que deux manifestations différentes d'un même processus physique à savoir le franchissement thermiquement activé d'obstacles par de petites portions de paroi indépendantes les unes des autres. Pour aller plus loin, il faut se donner un modèle de traînage de fluctuations. Nous avons utilisé celui de Street et Woolly [11] $\left({ }^{6}\right)$, approfondi par Gaunt [20], car il se prête bien au calcul.

Il s'agit d'un modèle formel qui représente un matériau ferromagnétique par un ensemble d'éléments de matière indépendants dont les retournements peuvent être thermiquement activés. On associe à cet ensemble une répartition $f(E)$ de barrières d'énergie $E$ qui dépendent à la fois des défauts et du champ magnétique appliqué. Le franchissement des barrières est gouverné par l'exponentielle de Boltzmann : la probabilité $\lambda$ de saut par unité de temps vaut $\lambda=1 / \tau=C \exp (-E / k T)$ où $C$ est de l'ordre de $10^{10} \mathrm{~s}^{-1}$ [11]. Le franchissement d'une barrière accroît en moyenne l'aimantation d'une quantité $i$. On trouve, tous calculs faits, que l'aimantation $I$ s'accroît à une vitesse :

$$
\frac{\mathrm{d} I(t, T)}{\mathrm{d} t}=i C \int_{E} f(E) \exp (-t / \tau) \exp (-E / k T) \mathrm{d} E .
$$

SW traitent les deux cas particuliers :

a) $f(E)=p$ pour $0<E<\infty$ (spectre plat infini). On trouve sans peine, aux temps qui sont accessibles expérimentalement $(C t \gg 1)$ :

$$
I(t, T)=\mathrm{Cte}+i p k T \log t
$$

ce qui permet de justifier l'allure logarithmique bien connue du traînage.

b) $f(E)=p$ pour $0<E<E_{0}$, 0 pour $E>E_{0}$ (créneau). SW expriment $I(t, T)$ sous forme d'un développement en série dont ils explicitent les deux premiers termes :

$$
I(t . T)=\mathrm{Cte}+i p k T\left(\log t-\hat{\lambda}_{0} t+\cdots\right)
$$

${ }^{(6)}$ Remarque : Nous noterons le modèle de Street et Woolly par SW. 
avec

$$
\lambda_{0}=\frac{1}{\tau_{0}}=C \exp \left(-E_{0} / k T\right)
$$

Nous avons complété ce calcul en obtenant une expression analytique valable à tout instant $t$.

Dans le cadre de ce modèle les deux types d'expériences s'identifient grâce à la définition d'une fenêtre d'analyse du spectre de barrière d'énergie.

Dans les deux types d'expériences, on fait une mesure physique à travers une fenêtre d'observation temporelle comprise entre deux instants $t_{1}$ et $t_{2}$ : dans le cas du traînage sous champ constant, $t_{1}$ vaut $1 \mathrm{~s}$ et $t_{2}, 25 \mathrm{~s}$; dans le cas du déplacement de la paroi à vitesse constante, $t_{1}=0,05 \mathrm{~s}$ est la limite expérimentale de résolution entre deux sauts successifs, $t_{2}$ est un peu plus délicat à déterminer. Nous le définirons comme le temps au bout duquel la paroi s'est déplacée d'une distance $d$ suffisante pour avoir oublié les obstacles auxquels elle était initialement sensible. Un choix raisonnable consiste à prendre $d=2000 \AA$. Compte tenu de la vitesse imposée à la paroi $\left(4000 \AA . \mathrm{s}^{-1}\right)$ $t_{2}$ vaut $0,5 \mathrm{~s}$.

On remarque dans les deux cas que seules certaines constantes de temps $\tau$ contribuent sensiblement aux phénomènes. En effet, les constantes de temps très courtes agissent avant l'instant $t_{1}$ où commence la mesure, alors qu'il faudrait attendre des temps supérieurs à $t_{2}$ pour observer la contribution des grandes constantes de temps. Soit $n_{0}$ le nombre initial de barrières d'énergie dont la constante de temps de franchissement est égale à $\tau$. Le nombre de ces barrières franchies par activation croît au cours du temps comme :

$$
n=n_{0}(1-\exp (-t / \tau))
$$

Pendant la mesure qui a lieu entre $t_{1}$ et $t_{2}$, on observe une variation de ce nombre :

$$
\Delta n=n_{2}-n_{1}=n_{0}\left(\exp \left(-t_{1} / \tau\right)-\exp \left(-t_{2} / \tau\right)\right) .
$$

$F(\tau)=\Delta n / n_{0}$ représente la fenêtre à travers laquelle on observe le spectre de barrières d'énergies. La constante de temps présentant l'efficacité maximum $\tau_{\mathrm{m}}$ a pour expression :

$$
\tau_{\mathrm{m}}=\frac{t_{2}-t_{1}}{\log t_{2} / t_{1}}
$$

A une température donnée $E$ et $\tau$ sont reliés par la relation :

$$
\frac{1}{\tau}=C \exp (-E / k T)
$$

où la quantité $C$ est a priori une fonction de la température. On peut montrer cependant que, en ce qui concerne nos expériences, on peut prendre $C$ indépendant de $T$ [21] et de l'ordre de $10^{10}$.
En résumé, une expérience à la température $T$ permet d'explorer le spectre des barrières d'énergie à travers une fenêtre dont la position et la largeur sont proportionnelles à $T$. $E_{\text {max }}$ et $\Delta E$ sont d'ailleurs très peu sensibles aux valeurs exactes de $t_{1}$ et $t_{2}$, à cause des variations très rapides de la quantité $1 / \tau$.

On montre d'abord que, dans le cas où $f(E)$ est une fonction puissance, le nombre de barrières vues à travers la fenêtre définie précédemment est de la forme : $N \sim T^{l+1}$; ce qui généralise le résultat de SW d'après lequel $N \sim T$ pour un spectre constant $\left(E^{0}\right)$.

La paroi ne s'accroche pas, à l'échelle des temps d'expérience, sur les obstacles correspondant à des barrières d'énergie trop faibles. Lorsqu'elle se détache d'un obstacle, le saut ainsi déclenché se propage jusqu'aux points d'ancrage les plus proches capables de l'arrêter. La rapide croissance de $v$ en fonction de $T$ montre que la densité de points d'ancrage efficaces diminue rapidement lorsque la température s'élève. Cette façon de voir est confirmée par la loi de distribution simple de volume des sauts :

$$
p(v) \sim \exp \left(-v / v_{0}\right) .
$$

Sawada [22] avait prévu une loi de ce type en admettant qu'il n'existe pas de corrélation entre la dureté des points d'ancrage successifs. A partir du volume moyen $v_{0}$ on peut définir un nombre $N$ d'accrochages efficaces inversement proportionnel à $v_{0}$, ce qui conduit pour $N$ à une loi du type $N \sim T^{-4,4}$ pour $T$ compris entre 253 et $323 \mathrm{~K}$. Dans cette même région de température $E \sim T^{-5,4}$.

La variation thermique du traînage de fluctuations thermiques $\Delta I_{1}^{25}$ (variation totale d'aimantation observée entre 1 et $25 \mathrm{~s}$ à la température $T$ ) présente une partie linéaire en $T$ aux basses températures, un maximum vers $30 \mathrm{~K}$ suivi d'une décroissance (cf. Fig. 34). La partie linéaire en $T$ s'interprète à l'aide

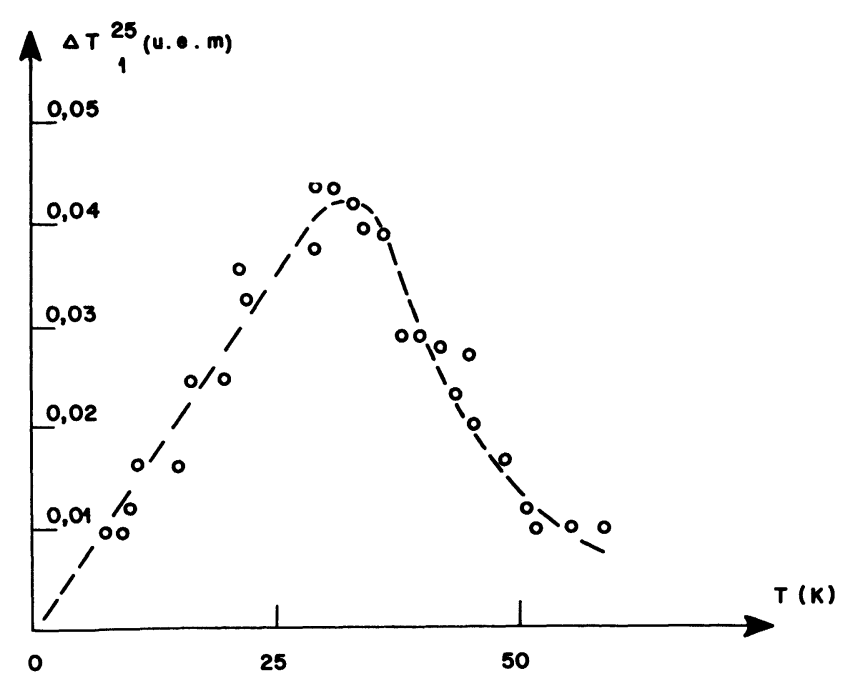

Fig. 34. - Variation d'aimantation entre $t=1 \mathrm{~s}$ et $t=25 \mathrm{~s}$ en fonction de la température.

[Change of magnetization between $t=1 \mathrm{~s}$ and $t=25 \mathrm{~s}$ plotted versus the temperature.] 
d'un spectre $f(E)$ ayant une région plate pour $E<E_{0}$. Pour avoir un ordre de grandeur de $E_{0}$ il suffit d'écrire que pour le maximum de $\Delta I_{1}^{25}$ la relation

$$
1 / \tau=C \exp (-E / k T)
$$

est satisfaite pour $\tau=\tau_{\mathrm{m}}=7,5 \mathrm{~s}, E=E_{0}$ et $T=30 \mathrm{~K}$. On trouve alors $E_{0} / k=630 \mathrm{~K}$. L'étape suivante consiste à trouver la forme du spectre pour $E>E_{0}$. On a vu que pour des énergies relativement élevées (5 $300<E / k<6800$ ) correspondant aux expériences du déplacement de la paroi à vitesse constante effectuées entre 253 et $323 \mathrm{~K}$, le spectre décroissait comme $E^{-5,4}$. Il n'y a pas de raison a priori pour que cette loi reste valable à des énergies plus basses; faisons cependant cette hypothèse. On doit alors observer au-delà de $E_{0}$ une décroissance en $E^{-5,4}$, ce qui se traduit par un traînage en $T^{-4,4}$. La figure 35 montre qu'on obtient ainsi un accord très raisonnable avec l'expérience. Pour $E_{0} / k=760 \mathrm{~K}$ on a : $\Delta I_{1}^{25} \sim T$ pour $0<T<30 \mathrm{~K} ; \Delta I_{1}^{25} \sim T^{-4,4}$ pour $T>36 \mathrm{~K}$.

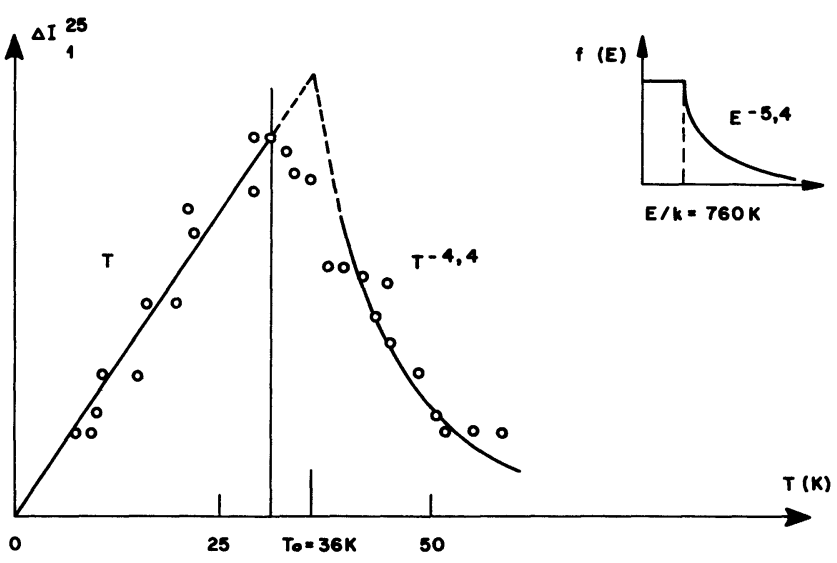

Fig. 35. - Comparaison des valeurs expérimentales du traînage avec la courbe calculée à partir du spectre d'énergie.

[Comparison between the experimental after-effect data and the curve calculated from the energy spectrum.]

Bien entendu, ces lois simples cessent d'être valables autour de $36 \mathrm{~K}$, lorsque la fenêtre d'analyse contient le point commun aux deux parties du spectre. Avec les critères que nous avons pris la région de raccordement se situe entre 31 et $40 \mathrm{~K}$. Il est également possible de préciser les valeurs numériques de $f(E)$. Le spectre de barrières d'énergie correspondant aux conditions expérimentales, c'est-à-dire pour un champ égal ou très voisin du champ coercitif, est tel que :

$$
\begin{gathered}
f(E)=10^{9} \text { pour } 0<E / k<760 \mathrm{~K} ; \\
4 \times 10^{24}(E / k)^{-5,4} \text { pour } E / k>760 \mathrm{~K} .
\end{gathered}
$$

La densité d'obstacles dans le cristal est également numériquement définie ; pour l'obtenir il suffit d'intégrer $f(E)$. Si on fait cette intégration entre 0 et l' $\infty$ on trouve un nombre total d'obstacles $N=10^{12}$ par $\mathrm{cm}^{3}$.
Nous allons maintenant discuter les résultats obtenus. Ce faisant, nous préciserons le rôle des fluctuations thermiques, certaines grandeurs physiques qui leur sont attachées, ainsi que des caractéristiques de la paroi (flexibilité) ou des imperfections du cristal (obstacles).

1) Les défauts du réseau cristallin de notre échantillon sont essentiellement des dislocations. Schultz [23] a récemment mesuré la force maximale qu'exerce une dislocation sur une paroi dans un grenat ferromagnétique : 0,62 dyne par $\mathrm{cm}$ de dislocation. L'ordre de grandeur n'est probablement pas très différent dans le $\mathrm{Fe}-\mathrm{Si}$. Pour estimer grossièrement l'énergie d'interaction correspondante supposons : que la longueur du segment de dislocation en interaction avec la paroi est de l'ordre de l'épaisseur de celle-ci, soit $2000 \AA$; que l'interaction subsiste sur un déplacement de paroi valant également $2000 \AA$.

On trouve alors une énergie d'interaction de $2,4 \times 10^{-10} \mathrm{erg}$, à comparer avec l'énergie d'activation typique $E_{0} / k=760 \mathrm{~K}$, soit $E_{0}=10^{-13} \mathrm{erg}$. L'apparente disproportion provient de ce que le champ appliqué au cours des expériences est voisin de $H_{\mathrm{c}}$ : il suffit pour faire franchir à la paroi les obstacles typiques qui s'opposent à son déplacement. Les fluctuations thermiques n'ont pas à fournir toute l'énergie nécessaire pour franchir les obstacles, comme ce serait le cas en champ nul, mais seulement une petite partie de celle-ci (cf. référence [24]).

2) Bien que secondaires sur le plan énergétique, les phénomènes d'activation thermique n'en déterminent pas moins les échelles de temps et d'espace caractéristiques des processus d'aimantation. La comparaison des densités d'obstacles effectifs à 0 et $300 \mathrm{~K}$ montre que, pour une paroi d'épaisseur $2000 \AA$, la distance moyenne entre obstacles actifs dans le plan de la paroi varie de quelques $\mu$ à quelques centaines de $\mu$. Il s'agit là d'un aspect essentiel trop souvent ignoré dans les modèles de déplacement de paroi.

3) Il est intéressant d'estimer certaines grandeurs physiques liées aux mécanismes d'activation thermique. Dans le modèle $S W$, la contribution élémentaire moyenne $i$ à l'aimantation est à relier au plus petit volume moyen $v_{\min }$ des sauts, que l'on observerait dans des expériences à très basse température lorsque tous les obstacles sont efficaces. Ce volume élémentaire vaut $v_{\text {min }}=1 / N_{\text {tot }}=10^{-12} \mathrm{~cm}^{3}$. Si l'on suppose que la flexibilité de la paroi est isotrope, et compte tenu de son épaisseur (environ $2000 \AA$ ), on trouve que la distance moyenne entre points d'ancrage dans le plan de la paroi serait voisine de $2 \mu$. En fait, les termes d'énergie magnétostatique (voir par exemple [25] et [26]) tendent à empêcher les modes de courbure de la paroi qui ne conservent pas le parallélisme entre ses génératrices et le vecteur aimantation. On doit donc considérer plutôt des déformations ayant l'allure de fuseaux allongés. Il est d'ailleurs difficile d'estimer le rapport entre les dimensions longitudinales et trans- 
versales de ces fuseaux. Un volume moyen par obstacle de $10^{-7} \mathrm{~cm}^{3}$ environ à la température ambiante correspond, pour un déplacement de $2000 \AA$, à une surface de paroi de $5 \times 10^{-3} \mathrm{~cm}^{2}$. Si on prend un coefficient d'allongement des fuseaux égal à 10 pour fixer les idées, on trouve que leurs dimensions transversales sont de l'ordre de $10 \mu$. Des expériences de dépolarisation de neutrons effectuées sur un échantillon similaire conduisent au même ordre de grandeur (M. Th. Rekveldt, communication personnelle).

4) Un autre modèle de traînage, proposé par Néel [27], évite justement d'introduire un spectre d'énergies d'activation. On suppose qu'on peut représenter l'effet des fluctuations thermiques par un champ fictif équivalent dont le calcul est d'ailleurs délicat. Les défauts et le champ appliqué n'interviennent dans ce modèle que d'un point de vue macroscopique, pour déterminer une susceptibilité irréversible. Pour faire le lien entre les deux aspects complémentaires d'énergie (SW) et le champ (Néel), on est obligatoirement conduit à définir un volume caractéristique ou volume d'activation $v^{*}$ à l'échelle duquel agissent les fluctuations. Pour estimer l'ordre de grandeur de $v^{*}$, écrivons [24] que l'énergie d'activation typique $E_{0}$ est égale à $2 I_{\mathrm{s}} v^{*} H$, où $I_{\mathrm{s}}=1700$ u.e.m. est l'aimantation à saturation, et $H \simeq H_{\mathrm{c}}=28 \mathrm{mOe}$. On trouve alors $v^{*}=10^{-15} \mathrm{~cm}^{3}$, soit le volume d'un cube dont l'arête serait égale à la moitié de l'épaisseur de paroi. Il y a donc une énorme disproportion $\left(10^{8}\right)$ entre le volume initial dans lequel se déclenche un saut et le volume final $v_{0}$ de ce saut à la température ambiante. Pour expliquer cette disproportion, nous avons admis que la déformation initiale de la paroi se propage en franchissant instantanément, à l'échelle des temps d'expérience, les obstacles trop faibles, et ne s'arrête que sur certains obstacles de dureté suffisante.

5) Néel suppose que les sauts thermiquement activés sont dus à l'effet des fluctuations thermiques intégré dans un certain volume caractéristique $v$, d'ailleurs assez délicat à définir. Il en déduit une expression du champ de fluctuation sous la forme

$$
h_{\mathrm{f}}^{2}=4 k T / 3 v \text {. }
$$

$\mathrm{Si}$ l'on prend pour $v$ le volume d'activation $v^{*}=10^{-15} \mathrm{~cm}^{3}$, on trouve à $300 \mathrm{~K} h_{\mathrm{f}}=13 \mathrm{Oe}$, valeur bien trop forte. Par contre, avec

$$
v=v_{0} \simeq 10^{-7} \mathrm{~cm}^{3}
$$

on trouve $h_{\mathrm{f}}=1,3 \mathrm{mOe}$, ce qui est beaucoup plus raisonnable : on sait en effet [28] que le champ de traînage est en règle générale une petite fraction du champ coercitif. Il semble donc bien, comme le suggère Néel, que le principal mécanisme du traînage soit l'action des champs magnétiques internes fluctuants produits par les oscillations thermiques de l'aimantation spontanée dans un volume relativement important à l'intérieur des domaines élémentaires. L'effet des oscil- lations thermiques de la paroi elle-même est vraisemblablement négligeable.

6) Nous avons employé volontairement dans cet article le terme d'obstacle pour représenter une région du cristal susceptible d'accrocher la paroi. L'essentiel des défauts dans le cristal est constitué par des dislocations isolées. Pfeffer $[29,30]$ a traité analytiquement le problème de l'interaction paroi-dislocation. Il a montré que l'interaction provient du réarrangement des moments magnétiques dans le champ de contraintes de la dislocation par l'intermédiaire du couplage magnétoélastique. Le potentiel d'interaction est de nature magnétostatique et, à cause de la décroissance en $1 / r$ du champ de contraintes, s'étend sur des distances relativement grandes : des observations optiques sur un monocristal de $\mathrm{YiG}$ [31] montrent que l'interaction se fait sentir jusqu'à une distance de $10 \mu$. De plus, la contrainte locale intervenant dans les équations sous forme quadratique, les différentes dislocations combinent leurs effets de façon non linéaire. Dès lors, nous considérons qu'il n'est pas possible d'attribuer l'accrochage d'une partie de la paroi à telle ou telle dislocation particulière et nous supposons simplement qu'on peut associer à chaque portion de la paroi se déplaçant de façon cohérente une énergie potentielle qui sera une fonction aléatoire des coordonnées d'espace, et non d'une coordonnée d'espace comme dans le cas de la paroi rigide. Nous employons le terme d'obstacles pour désigner les maxima locaux de ce potentiel. On trouve une approche analogue dans la référence [24]. L'énergie superficielle de la paroi limite sa flexibilité. Une telle approche ne peut être cohérente que si elle conduit à des dimensions suffisantes pour les portions de paroi mise en jeu. Celles-ci doivent être toujours supérieures à l'épaisseur de paroi, soit $2000 \AA$. On mesure ainsi la difficulté d'une approche théorique du problème. Une première étape consiste à définir l'interaction d'une paroi avec une dislocation isolée par exemple. Ce point est encore imparfaitement résolu si l'on en juge par des résultats récents [23] : la théorie prévoit que la force exercée par une dislocation coin passe par un maximum lorsque la direction de la force est perpendiculaire au vecteur de Burgers. L'expérience révèle par contre deux pics très nets; il y a donc désaccord qualitatif. Dans une deuxième étape, il faut rechercher l'effet d'une distribution de défauts, et pour cela, résoudre un problème non linéaire.

7. Conclusion. - Ce travail avait essentiellement pour but l'étude des processus d'aimantation. Il montre que même dans un cas aussi simple que celui d'une seule paroi de Bloch à $180^{\circ}$ se déplaçant dans un milieu peu perturbé, les mécanismes réels ont une certaine complexité. Il souligne en particulier le rôle prépondérant de l'agitation thermique que l'on néglige habituellement. Des méthodes statistiques simples appliquées aux séquences de sauts Barkhausen nous ont permis de tirer des conclusions physiques intéres- 
santes sur les mécanismes d'aimantation. Mais, quoique le bruit Barkhausen soit toujours le reflet de ces mécanismes, il nous paraît extrêmement difficile, dans un cas plus complexe comme celui d'un polycristal, de remonter de ses caractéristiques aux causes qui l'ont produit. Il nous semble sur deux points au moins qu'il soit possible d'utiliser des méthodes statistiques plus élaborées que celles que nous avons employées.

Le premier est la recherche d'une ressemblance entre séquences de sauts correspondant à des déplacements de la paroi d'amplitude de plus en plus grande. On pourrait ainsi avoir des informations sur la manière dont on perd la propriété de conservativité. Le second est l'élaboration d'un modèle statistique des séquences de sauts Barkhausen à partir des résultats expérimentaux relatifs au modèle de la fonction potentiel. L'aspect de filtrage développé par Bonnet [5] nous semble un point de départ original qui permet l'introduction naturelle de la théorie des fonctions aléatoires. Il mérite d'être approfondi. Dans une deuxième étape, on pourrait essayer d'inclure dans le modèle l'influence de l'agitation thermique qui est prépondérante et de caractère aléatoire. L'autre point de départ possible est celui développé au paragraphe 5 . Il ne faut également pas perdre de vue que l'on a affaire à un système non linéaire, hors d'équilibre et doué d'hystérésis. Cet aspect nous a permis d'interpréter les avalanches de sauts de Barkhausen que l'on observe au voisinage du champ coercitif dans des matériaux polycristallins tels que le fer et l'acier. Nous avons montré que le passage d'un régime de déplacement par morceaux à un déplacement en bloc de la paroi avait le caractère d'une instabilité : il se produit grâce à un couplage progressif entre événements élémentaires initialement indépendants. D'autre part, quand on fait décrire à la paroi des cycles d'hystérésis successifs de faible amplitude, on observe une évolution du cycle analogue au phénomène de reptation d'un polycristal. Ce dernier peut être considéré comme un révélateur

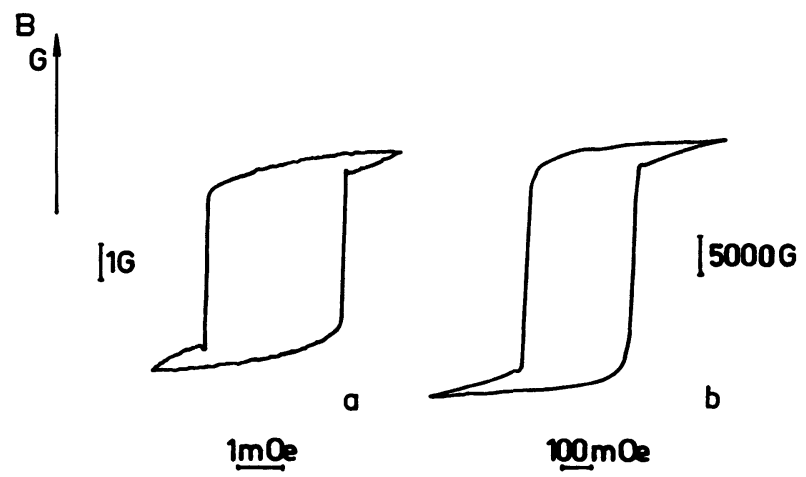

moe

Fig. 36. - a) Cycle d'hystérésis d'une paroi unique. b) Cycle d'hystérésis de l'échantillon de fer pur polycristallin.

(a) Hysteresis loop of a single wall. b) Hysteresis loop of the polycrystalline sample of pure iron.] de l'existence du caractère collectif des déplacements de paroi dans le polycristal. La figure 36 permet de comparer le cycle d'hystérésis élémentaire relatif à une paroi de Bloch dans le monocristal de Fe-Si étudié ici et le cycle d'hystérésis d'un polycristal de fer pur. Leur allure générale est très comparable. On remarquera les nombreuses similitudes entre le comportement de la paroi unique et du polycristal qui sont vraisemblablement dues au fait que l'on a dans chacun des deux cas apparition de phénomènes collectifs résultant de couplages entre éléments non linéaires. La différence essentielle est ici l'échelle à laquelle se produisent les phénomènes. Partant de l'idée que le déplacement d'une paroi de Bloch dans un polycristal peut rester isolé ou au contraire déclencher une avalanche de sauts dans une structure en domaines, on peut expliquer quantitativement l'allure de la courbe de première aimantation ainsi que les cycles de reculs de divers matériaux [18], dans un domaine de champs où l'aimantation évolue grâce à la modification de la structure en domaines élémentaires. Ce modèle donne le nombre moyen de sauts dans une avalanche en fonction du champ. L'accord entre les déterminations directes résultant de l'étude du bruit de Barkhausen sur des matériaux du même type est satisfaisant.

Ces divers points nous semblent constituer une amorce intéressante pour un travail pluridisciplinaire dans lequel les méthodes statistiques seraient autre chose qu'un outil pour l'étude des mécanismes d'aimantation. Un tel travail doit être le fruit d'une étroite collaboration entre les spécialistes des phénomènes aléatoires et du magnétisme.

Remerciements. - Une part importante des travaux des auteurs cités dans cet article n'aurait pas été possible sans une étroite collaboration entre le laboratoire de Magnétisme et le CEN-G (L.ETI, M.C.T.E. Division Traitement du Signal). Les auteurs tiennent à remercier particulièrement $M$. Jacques Max qui a su la rendre agréable et efficace.

\section{ANNEXE}

Modèle des ressorts. - Ce modèle est dû à J. A. Baldwin (cf. J. Appl. Phys. 42 (1971) 1963) ; c'est un exemple de modèle non conservatif.

On représente l'interaction paroi défaut par un ressort qui s'attache à la paroi lorsqu'elle rencontre le défaut. La force de rappel du ressort est du type $-k x$; pour un allongement donné $x_{0}$, le ressort casse et le défaut n'exerce plus aucune force sur la paroi. On introduit ainsi du point de vue phénoménologique, des termes réversibles (allongements) et irréversibles (cassures). On tient compte de la statistique des défauts grâce à une distribution $n\left(x_{0}\right)$ des allongements pour lesquels les ressorts cassent. Dans les champs faibles on retrouve ainsi les lois de Ray- 
leigh : les déplacements réversibles moyens sont proportionnels à $H$, alors que les déplacements irréversibles conduisent à un terme en $H^{2}$. Les hypothèses physiques sont très différentes du modèle de Néel. Dans ce dernier, la paroi voit la même fonction potentiel $V(x)$ lors de deux passages successifs entre deux valeurs $x_{1}$ et $x_{2}$ du déplacement : c'est la propriété de conservativité. Il n'en est plus de même dans le modèle de Baldwin. Lors d'un aller et retour d'une abscisse $x_{1}$ à $x_{2}$ certains ressorts se sont cassés à l'aller et d'autres se sont connectés au retour et l'énergie potentielle stockée dans le système de ressort est différente. C'est un modèle non conservatif. Du point de vue physique cela signifie que l'on tient compte de la flexibilité de la paroi : en effet, on peut avoir des configurations différentes, donc des énergies différentes pour une même position du plan moyen.

\section{Bibliographie}

[1] Kitrel, C., Physical theory of ferromagnetic domains, Rev. Mod. Phys. 21 (1949). Cet exposé, considéré aujourd'hui comme classique, a été reproduit dans de nombreux ouvrages en particulier :

KrTtel, C., Introduction à la physique de l'état solide (Dunod, edit.) 1970

ChiKazumi, S., Physics of Magnetism (J. Wiley) 1964.

[2] Brissonneau, P., Les domaines magnétiques, Revue Phys. Appl. 9 (1974).

[3] Néel, L., Cah. Phys. 12 (1942), Cah. Phys. 13 (1943).

[4] Lord Rayleigh, Philos. Mag. 23 (1887) 225.

[5] Bonnet, G., J. Physique 28 (1967).

[6] Vergne, R., Porteseil, J. L., Revue Phys. Appl. 6 (1971) 95.

[7] Porteseil, J. L., Vergne, R., J. Physique 37 (1976) 1211.

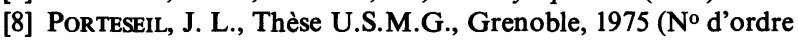
CNRS A.O. 11350).

[9] Porteseil, J. L., Physica 93 B (1978) 201.

[10] Ferrari, G., Porteseil, J. L., Vergne, R., Intermag (1978), Florence. IEEE Trans. Magn. 14, 5 (1978) 764.

[11] Street, R., Woolly, J. C., Proc. Phys. Soc. A62 (1949) 562.

[12] Néel, L., C.R. Hebd. Séan. Acad. Sci. 244 (1957) 2688.

[13] Porteseil, J. L., Vergne, R., Revue Phys. Appl. 12 (1977) 1077.
[14] DaNG, N. V., Thèse Grenoble (1961).

[15] Rogalla, H., Heiden, C., Phys. Lett. 63 A (1977) 63.

[16] Grosse-NobIS, W., J. Magn. Magn. Mater. 4 (1977) 247.

[17] MandelBrot, B., Les objectifs fractals : forme, hasard et dimensions, (Flammarion, Paris) 1975, p. 51 à 63.

[18] Porteseil, J. L. et Vergne, R., J. Phys. 40 (1979) 67.

[19] Porteseil, J. L., Cotillard, J. C., Vergne, R. et Ferrari, G., « Etude expérimentale des mécanismes d'activation thermique dans le déplacement d'une paroi de Bloch à $180^{\circ}$ " J. Physique 42, septembre (1981).

[20] Gaunt, P., Philos. Mag. 34 (1976) 775.

[21] Barbara, B., Uehara, M., Physica 86-88 B (1977) 1481.

[22] Sawada, H., J. Phys. Soc. Jpn 7 (1952) 564.

[23] Schultz, L., J. Magn. Magn. Mater. 13 (1979) 251.

[24] Siemers, D., Nembach, E., Phys. Lett. 65 A (1978) 345.

[25] Dietze, H. D., Z. Phys. 149 (1957) 276.

[26] MARKERT, H., Z. Naturfors. 29 a (1974) 457.

[27] NeEl, L., J. Physique Radium 12 (1951) 339.

[28] Barbier, J. C., Thèse, Université de Grenoble (1953).

[29] Pfeffer, K. H., Phys. Status Solidi 20 (1967) 395.

[30] Pfeffer, K. H., Phys. Status Solidi 21 (1967) 837.

[31] Vlasko-Vlasov, V. K., Dedukh, L. M., Nikitenko, V. I., Sov. Phys. JETP 38 (1974) 184.

[32] Porteseil, J. L., Phys. Status Solidi (a) 51 (1979) 107. 